\title{
Extremely high velocity gas from the massive young stellar objects in IRAS 17233-3606
}

\author{
S. Leurini ${ }^{1}$, C. Codella ${ }^{2}$, L. A. Zapata ${ }^{3}$, A. Belloche ${ }^{3}$, T. Stanke ${ }^{1}$, F. Wyrowski ${ }^{3}$, P. Schilke ${ }^{3,4}$, \\ K. M. Menten ${ }^{3}$, and R. Güsten ${ }^{3}$
}

1 ESO, Karl-Schwarzschild Strasse 2, 85748 Garching-bei-München, Germany e-mail: sleurini@eso.org

2 INAF, Osservatorio Astrofisico di Arcetri, Largo E. Fermi 5, 50125 Firenze, Italy

Max-Planck-Institut für Radioastronomie, Auf dem Hügel 69, 53121 Bonn, Germany

4 Physikalisches Institut, Universität zu Köln, Zülpicher Str. 77, 50937 Köln, Germany

Received 29 June 2009 / Accepted 21 August 2009

\section{ABSTRACT}

\begin{abstract}
Context. Molecular outflows from high-mass young stellar objects provide an excellent way to study the star formation process, and investigate if they are scaled-up versions of their low-mass counterparts.

Aims. We selected the nearby massive star forming region IRAS 17233-3606 in order to study the kinematics and physics along the molecular outflow(s) originating from this source.

Methods. We observed IRAS 17233-3606 in CO, a typical tracer of gas associated with molecular outflow, with the Submillimeter Array in the (2-1) transition, and with the APEX telescope in the higher excitation (6-5) line. Additional infrared $\mathrm{H}_{2}$ observations were performed with the UKIRT telescope. The CO data were analysed using a LVG approach.

Results. Our data resolve the previously detected molecular outflow into at least three different components, one of them with a high collimation factor $(\sim 4)$, and characterised by emission at extremely high velocities $\left(\left|v-v_{\text {LSR }}\right|>120 \mathrm{~km} \mathrm{~s}^{-1}\right)$. The estimate of the kinematical outflow parameters are typical of massive YSOs, and in agreement with the measured bolometric luminosity of the source. The kinematic ages of the flows are in the range $10^{2}-10^{3} \mathrm{yr}$, and therefore point to young objects that still have not reached the main sequence.
\end{abstract}

Key words. ISM: jets and outflows - ISM: molecules - stars: individual: IRAS 17233-3606 - stars: formation

\section{Introduction}

Although massive stars are fundamental for astrophysics, little is known of their earliest evolutionary phases. The main problem for the understanding of massive star formation comes from the radiation pressure that massive stars exert on their surrounding medium once they have reached a mass of around $8 M_{\odot}$, that should prevent further accretion onto the star. As a consequence, stars with mass larger than $8 M_{\odot}$ should not exist (Palla \& Stahler 1993), in clear disagreement with observations. Two main theoretical scenarios have been proposed to solve this problem: (1) accretion as in the low-mass case through more massive circumstellar disks and/or sufficiently high accretion rates (e.g., Yorke \& Sonnhalter 2002) and (2) coalescence of lowermass stars belonging to the cluster that harbours the high-mass star(s) (Bonnell et al. 1998; Bonnell \& Bate 2002). One of the fundamental observational tests to distinguish between the different proposed models is to detect collimated outflows and accretion disk from massive young stellar objects (YSOs), and to assess the fraction of still embedded massive YSOs with disks (Cesaroni et al. 2007; Krumholz \& Bonnell 2007). Since massive pre-main sequence stars may lose any disk they originally had due to the radiation field (e.g., Natta et al. 2000; Fuente et al. 2003), such studies are best done in earlier evolutionary phases. However, large distances and a high degree of multiplicity among early spectral type stars (e.g., Preibisch et al. 2001) make observations of these phases of massive YSOs a challenge, especially at (sub)mm wavelengths where current facilities still lack the necessary resolution to resolve single massive objects at such distances.

Despite the observational effort in the last decade to investigate the early evolutionary phases of massive stars, only few sources show typical phenomena associated with low-mass star formation such as collimated jets and outflows, circumstellar disks even at high angular resolution (Beuther \& Shepherd 2005; Arce et al. 2007; Cesaroni et al. 2007). In addition, neither collimated jet-like outflows or circumstellar disks have been detected in sources more massive than early B- and late O-type YSOs, although recently Zapata et al. (2009) detected a relatively collimated outflow $\left(14^{\circ}\right)$ towards W51 IRS2 and suggested the presence of a Keplerian infalling ring around a central object of at least $60 M_{\odot}$.

It is still not clear whether the lack of detection of collimated outflows and circumstellar disks around O-type YSOs is an observational bias due to the poor angular resolution and sensitivity of the observations or whether it reflects a real difference in the way massive stars form. Beuther \& Shepherd (2005) suggested an evolutionary scenario in which the collimation of the outflows decreases with time, due to the interaction with the wind from the central object. Hence, the need to investigate high-mass YSOs in different evolutionary stages and of different spectral types at high linear resolution is clear.

The region harbouring the prominent far-infrared source IRAS 17233-3606 (hereafter IRAS 17233) is one of the best laboratories in which to attack the problem of massive star formation. IRAS 17233 first came to attention through its very 


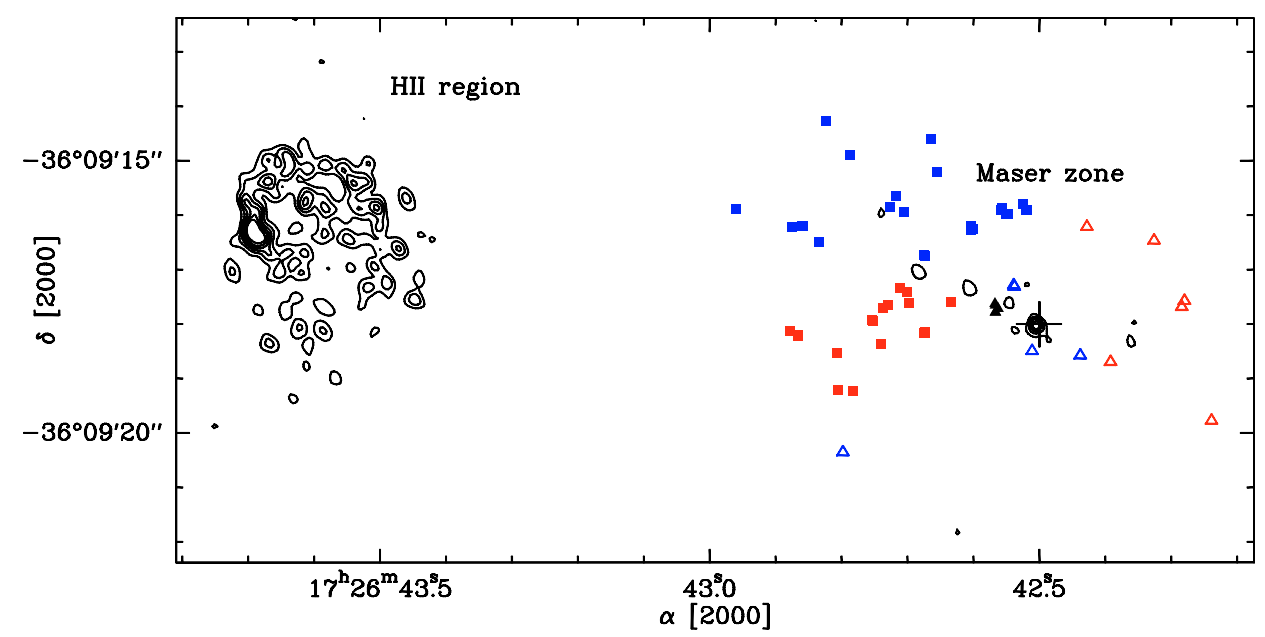

Fig. 1. Overview of the region: the black contours show the $1.3 \mathrm{~cm}$ continuum emission of IRAS 17233 (Zapata et al. 2008), from $0.12 \mathrm{Jy} \mathrm{beam}^{-1}(3 \sigma)$ in step of $0.24 \mathrm{Jy} \mathrm{beam}^{-1}$. The HII region and the maser zone are labelled. Overlaid on the map are the positions of the $\mathrm{OH}$ (blue and red squares, Fish et al. 2005), $\mathrm{CH}_{3} \mathrm{OH}$ (black triangles, Walsh et al. 1998) and $\mathrm{H}_{2} \mathrm{O}$ (blue and red open triangles, Zapata et al. 2008) masers. The cross marks the position of the peak of the SMA $1.3 \mathrm{~mm}$ continuum emission, used as reference for the APEX observations.

intense $\mathrm{H}_{2} \mathrm{O}, \mathrm{OH}$, and $\mathrm{CH}_{3} \mathrm{OH}$ masers (e.g., Caswell et al. 1980; Fix et al. 1982; Menten 1991). The luminosity of IRAS 17233 ranges between $1.4 \times 10^{5} L_{\odot}(d=2.2 \mathrm{kpc}$, Hughes \& MacLeod 1993 , based on the IRAS colours $)$ and $1.7 \times 10^{4} L_{\odot}(d=0.8 \mathrm{kpc}$, Faúndez et al. 2004, obtained by integrating the spectral energy distribution). Previous studies seem to agree that IRAS 17233 is located at the near kinematic distance (between $700 \mathrm{pc}$ and $2.2 \mathrm{kpc}$, Miettinen et al. 2006; Forster \& Caswell 1989) rather than at the far distance ( $\sim 16 \mathrm{kpc}$, e.g. MacLeod et al. 1998). This is suggested by the high measured intensities of continuum and line emission at practically all the observed wavelengths, which would indicate exceedingly high luminosities if the source were at the far distance and, persuasively, by the fact that it is at an angular distance of more than 0.5 degrees below the Galactic plane. Moreover, the source lies at the edge of an infrared dark cloud (Leurini et al., in prep.). In the following analysis, we assumed a distance of $1 \mathrm{kpc}$ for IRAS 17233.

Recently, high angular resolution multi-radio wavelengths observations by Zapata et al. (2008) resolved a cluster of nine compact radio sources of different nature in the region. Of these objects, one corresponds to the HII region already mapped at $\mathrm{cm}$-wavelengths by different authors (e.g., Hughes \& MacLeod 1993; Walsh et al. 1998), while four others are found close to the maser zone (i.e., the area of the region where the $\mathrm{H}_{2} \mathrm{O}, \mathrm{CH}_{3} \mathrm{OH}$ and $\mathrm{OH}$ masers are detected, see Fig. 1). Zapata et al. (2008) also found that one of these four sources (labelled VLA 2d in their paper) is at the centre of a bipolar north-south outflow detected by Fish et al. (2005) in OH masers. Zapata et al. (2008) also reported the presence of a ring-like structure traced by $\mathrm{H}_{2} \mathrm{O}$ masers, which they interpret as due to northeast-southwest outflows. Leurini et al. (2008) reported the discovery of a bipolar outflow originating from the vicinity of IRAS 17233, through low resolution observations performed in $\mathrm{CO}(3-2)$ with the APEX telescope $\left(\sim 18^{\prime \prime}\right.$ resolution). From these data however, the powering source of the molecular outflow remains unidentified. Leurini et al. (2008) also reported a rich molecular spectrum from a position very close to the centre of the outflow, typical of hot molecular cores near massive young stellar objects, that represent the stage of massive star formation when the newly formed star is efficiently heating the surrounding medium up to hundreds of $\mathrm{K}$, but it has not yet developed an ionised region (e.g., Kurtz et al. 2000; Cesaroni et al. 2003).
In this paper, we present observations of the molecular outflow in IRAS 17233 at 220 and $230 \mathrm{GHz}$ in ${ }^{13} \mathrm{CO}(2-1)$ and $\mathrm{CO}(2-1)$ with the Submillimeter Array (SMA), and at $690 \mathrm{GHz}$ in $\mathrm{CO}(6-5)$ with the APEX telescope ${ }^{1}$. The corresponding continuum data of the SMA observations, as well as the other spectral line data, will be presented in a forthcoming paper. While the SMA $\mathrm{CO}(2-1)$ data allow us to reach a high angular resolution (see Table 1) and study the outflow in its detail, the APEX $\mathrm{CO}(6-5)$ observations trace the warm component of the outflow, and allow a reliable estimate of its excitation conditions. Despite the importance of observations of high rotational $\mathrm{CO}$ transitions in the study of molecular outflows, only a handful of sources have been mapped in rotational CO lines with J higher than 3 (e.g., Beuther et al. 2002a; Leurini et al. 2006; van Kempen et al. 2009) because of the low transmission of the atmosphere at these frequencies.

\section{Observations}

\subsection{SMA observations}

We observed IRAS 17233 with the SMA interferometer on April 10, 2007 in the compact configuration with seven antennas. The receivers operated in a double-sideband mode with an IF band of 4-6 GHz so that the upper and lower sideband were separated by $10 \mathrm{GHz}$. The central frequencies of the upper and lower sideband were 220.4 and $230.4 \mathrm{GHz}$ respectively, allowing to detect the ${ }^{13} \mathrm{CO}(2-1), \mathrm{C}^{18} \mathrm{O}(2-1)$ and $\mathrm{SO}\left(5_{6}-4_{5}\right)$ lines in the lower side band of the receivers, the $\mathrm{CO}(2-1)$ transition in the upper side band. The correlator had a bandwidth of $\sim 1.9 \mathrm{GHz}$ and the channel spacing was $0.406 \mathrm{MHz}$, corresponding at this frequency to a velocity resolution of $0.5 \mathrm{~km} \mathrm{~s}^{-1}$. The adopted systemic velocity was $-3.4 \mathrm{~km} \mathrm{~s}^{-1}$. The observations were performed under good weather conditions with zenith opacities $\tau(225 \mathrm{GHz})$ between 0.1 and 0.14 measured by the National Radio Astronomy Observatory (NRAO) tipping radiometer operated by the Caltech Submillimeter Observatory (CSO).

1 This publication is based on data acquired with the Atacama Pathfinder Experiment (APEX). APEX is a collaboration between the Max-Planck-Institut für Radioastronomie, the European Southern Observatory, and the Onsala Space Observatory. 
Table 1. Observational parameters

\begin{tabular}{ccccc}
\hline \hline Transition & $\begin{array}{c}\text { Frequency } \\
(\mathrm{GHz})\end{array}$ & $\begin{array}{c}H P B W \\
\left({ }^{\prime \prime}\right)\end{array}$ & $\begin{array}{c}\text { PA } \\
\left({ }^{\circ}\right)\end{array}$ & $\begin{array}{c}\Delta v \\
\left(\mathrm{~km} \mathrm{~s}^{-1}\right)\end{array}$ \\
\hline${ }^{13} \mathrm{CO}(2-1)$ & 220.3987 & $5.43 \times 1.88$ & 30 & 0.50 \\
$\mathrm{CO}(2-1)$ & 230.5380 & $5.45 \times 2.08$ & 28 & 0.50 \\
$\mathrm{CO}(6-5)$ & 691.4731 & 8.9 & - & 0.05 \\
\hline
\end{tabular}

We covered the molecular outflow from IRAS 17233 presented by Leurini et al. (2008) with a mosaic of two fields centred at $\alpha_{2000}=17^{\mathrm{h}} 26^{\mathrm{m}} 41^{\mathrm{s}} .76, \delta_{2000}=-36^{\circ} 09^{\prime} 00^{\prime} 5$ and $\alpha_{2000}=17^{\mathrm{h}} 26^{\mathrm{m}} 42^{\mathrm{s}} .4, \delta_{2000}=-36^{\circ} 09^{\prime} 10^{\prime} .5$. The projected baselines ranged between 5 and $88 \mathrm{~m}$. The short baseline cutoff implies that source structures $\geq 54^{\prime \prime}$ are filtered out by the observations. However, intermediate structures are also affected by missing fluxes.

Bandpass calibration was done with 3 C 454.3. We used Callisto for the flux calibration which is estimated to be accurate within $20 \%$. Gain calibration was done via frequent observations of the quasars 1626-298 and 1713-269. Measured double-side band system temperatures corrected to the top of the atmosphere were between 100 and $400 \mathrm{~K}$, mainly depending on the elevation of the source. Details on the observational setup are given in Table 1.

The initial flagging and calibration was done with the IDL superset MIR originally developed for the Owens Valley Radio Observatory and adapted for the SMA ${ }^{2}$. The imaging and data analysis was conducted in MIRIAD (Sault et al. 1995) and MAPPING $^{3}$. Images were produced using natural weighting. The resulting synthesised beams are listed in Table 1 . The high degree of elongation of the beam is due to the low elevation of the source from Hawaii. However, the spatial resolution is highest along the direction perpendicular to the outflow, making the dataset well-suited to study its morphology and kinematics. In order to compare the SMA observations to the APEX data, we resampled the final data to a spectral resolution of $2 \mathrm{~km} \mathrm{~s}^{-1}$; the corresponding rms is $0.05 \mathrm{Jy} / \mathrm{beam}$.

\subsection{APEX observations}

IRAS 17233 was observed in the $\mathrm{CO}(6-5)$ transition with the $\mathrm{CHAMP}^{+}$dual-frequency heterodyne submillimetre array receiver on APEX. The observations were performed on 2007, October 20 and 22, under good weather conditions (with 0.9 and $0.7 \mathrm{~mm}$ precipitable water vapour). The peak of the SMA continuum emission $\left(\alpha_{\mathrm{J} 2000}=17^{\mathrm{h}} 26^{\mathrm{m}} 42.455^{\mathrm{s}}\right.$, $\left.\delta_{\mathrm{J} 2000}=-36^{\circ} 09^{\prime} 18.047^{\prime \prime}\right)$ was used as reference position. The system temperature was between 4500 and $14000 \mathrm{~K}$ on October 20th, between 2800 and $4500 \mathrm{~K}$ on October 22nd. Pointing was established by total power continuum cross-scans on SgrB2(N), and found to be accurate within $\sim 3^{\prime \prime}$.

The beam efficiencies of $\mathrm{CHAMP}^{+}$were determined via observations of Mars and Jupiter, the first at $691 \mathrm{GHz}$, the second at $661 \mathrm{GHz}$, and found to be 0.38 and 0.45 , respectively. We therefore adopted a beam efficiency of 0.40 to convert from antenna temperature into main-beam temperature units. The original spectral resolution of the data $\left(0.05 \mathrm{~km} \mathrm{~s}^{-1}\right)$ was smoothed to $2 \mathrm{~km} \mathrm{~s}^{-1}$ for our analysis. The rms noise in the final data cube is not uniform over the channels, because the northern part of

\footnotetext{
2 The MIR cookbook by Charlie Qi can be found at http: //cfa-www. harvard. edu/ cqi/mircook.html.

3 http://www.iram. fr/IRAMFR/GILDAS
}

the map was observed only on October 20, and because of the different weather conditions during the two days. The rms noise is of the order $1-4 \mathrm{~K}$, larger on the northern part of the map. The maps were produced with the XY_MAP task of CLASS90, which convolves the data with a Gaussian of one third of the beam: the final angular resolution of the data is 9.' 4 .

\subsection{UKIRT observations}

We obtained near-infrared wide field images using WFCAM on UKIRT through $J H K$ broad band and a $\mathrm{H}_{2}$ narrow band filter on May 3rd and 29, 2008. Integration times were $3.75 \mathrm{~min}$ for the broad band filters and 24 min for the narrow band filter. We used the data as reduced through the CASU pipeline. We used the $K$ broad band image to discriminate between continuum and emission line features in the $\mathrm{H}_{2}$ narrow band image. We created a continuum subtracted, pure $\mathrm{H}_{2}$ emission line image by properly registering the broad and narrow band images and smoothing the narrow band image to match the poorer seeing of the broad band image. We derived a flux scaling factor by comparing the counts of a number of stars in both filters and subtracted the scaled broad band image from the narrow band image. The resulting emission line image reveals a number of jet-like features, of which we will only discuss the counterparts of the $\mathrm{CO}$ outflows here. The other flows from the cluster forming core and along the filamentary dark cloud will be presented elsewhere (Stanke et al., in prep.).

\section{Observational results}

\subsection{CO emission}

Our new observations significantly improve the angular resolution of the previous study (Leurini et al. 2008), revealing the structure of the molecular outflow in detail. The $\mathrm{CO}$ transitions are characterised by emission up to extremely high velocities in the red- and blue-shifted wings. We adopt here a systemic velocity of $-3.4 \mathrm{~km} \mathrm{~s}^{-1}$ for the source (Bronfman et al. 1996), which is confirmed by spectral features in the SMA dataset with peak around $-3.0 \mathrm{~km} \mathrm{~s}^{-1}$. The blue-shifted emission extends to $\sim-200 \mathrm{~km} \mathrm{~s}^{-1}$ in the $\mathrm{CO}(2-1)$ line, and to $\sim-80 \mathrm{~km} \mathrm{~s}^{-1}$ in the $\mathrm{CO}(6-5)$ transition. The red-shifted lobe extends to velocities up to $120 \mathrm{~km} \mathrm{~s}^{-1}$ in $\mathrm{CO}(2-1)$, to $\sim 60 \mathrm{~km} \mathrm{~s}^{-1}$ in the (6-5) line. In Fig. 2, we show the $\mathrm{CO}$ spectra towards the peak of the $1.3 \mathrm{~mm}$ continuum emission.

Based on the channel maps, we identify two different velocity regimes in the $\mathrm{CO}(2-1)$ emission: the extremely high velocity regime (EHV: blue: $-200<v_{\text {LSR }}<-130 \mathrm{~km} \mathrm{~s}^{-1}$; red: $90<v_{\mathrm{LSR}}<120 \mathrm{~km} \mathrm{~s}^{-1}$ ), and the high velocity regime ( $\mathrm{HV}$ : blue: $-130<v_{\mathrm{LSR}}<-25 \mathrm{~km} \mathrm{~s}^{-1}$; red: $16<v_{\mathrm{LSR}}<50 \mathrm{~km} \mathrm{~s}^{-1}$ ). The integrated intensity in the two velocity intervals is presented in the left panel of Fig. 3. For the high velocity red-shifted emission, we did not integrate over the velocities $(50,90) \mathrm{km} \mathrm{s}^{-1}$ because of contamination from other molecular species at the peak of the mm continuum emission (see Fig. 2). The molecular spectrum towards the peak of the continuum emission is extremely rich and typical of a hot-core, with very few channels free of line emission. For this reason, we cannot exclude contamination from other molecular species, but this problem should affect our maps only at the centre of the mm continuum emission. The platforming producing an artificial negative continuum emission, seen in the spectra towards this position (Fig. 2), is probably a result of at least two effects: first, it is not unlikely that we overestimate the contribution of the continuum, since at the position 


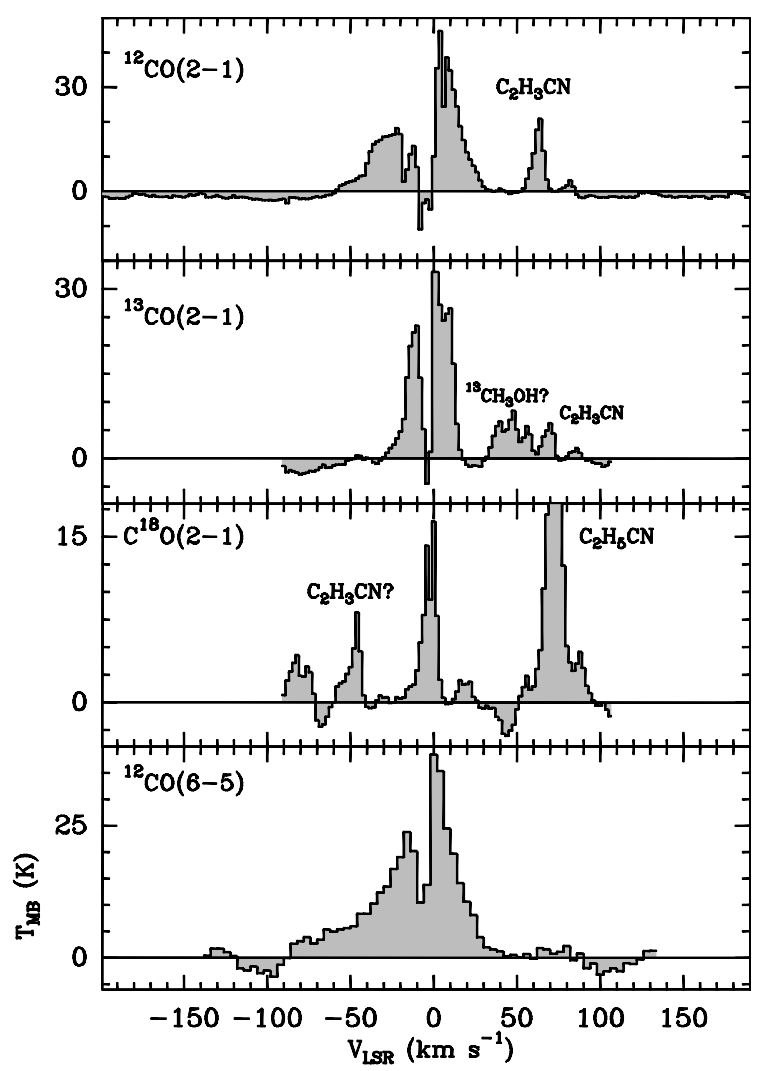

Fig. 2. Spectra of CO and its isotopologues at the peak of the mm continuum emission; the spectral features due to different molecular species are marked.

of the hot core the density of lines is extremely high and it is difficult to define channels free of line emission. Since the platforming affects only the area of the map where the continuum emission is detected (see Figs. 4 and 5 for comparison of spectra at other positions), we believe this to be the most likely reason for it. However, higher energy transition lines (e.g., $\mathrm{CO}(6-5)$ but also $\mathrm{CO}(3-2)$, Leurini et al. 2008) have wing emission at very high velocities, and therefore it is possible that large structure emission is filtered out by the SMA and results in negative features.

The SMA CO maps reveal a clumpy structure with well separated blue- and red-shifted emission, and an overall structure well aligned along the $\mathrm{N}-\mathrm{S}$ direction and centred close to the peak of the mm continuum emission $\left(\alpha_{\mathrm{J} 2000}=17^{\mathrm{h}} 26^{\mathrm{m}} 42.455^{\mathrm{s}}\right.$, $\delta_{\mathrm{J} 2000}=-36^{\circ} 09^{\prime} 18^{\prime \prime} .047$, see Fig. 6). This peak is associated with the maser zone and not with the HII region reported by several authors (see Fig. 1). The red-shifted emission shows four peaks: R1 associated with HV and EHV emission, R2 and R3 only with HV gas, and R4 with EHV emission only. Similarly, the blue-shifted emission peaks at four positions: B1 and B2, associated only with HV gas, and B3 and B4 associated with EHV emission. The spectra at the eight peaks of the $\mathrm{CO}(2-1)$ emission are shown in Figs. 4 and 5.

Figure 3 also shows the integrated emission of the blueand red-shifted wings of the $\mathrm{CO}(6-5)$ line. Despite the different resolutions, the two maps are very similar. Emission is detected at all positions detected in $\mathrm{CO}(2-1)$, except on the highvelocity B4.

To roughly quantify the degree of collimation of the whole outflow structure as traced by $\mathrm{CO}(2-1)$, we divided the length of the outflow by its width, which results in a collimation factor $f_{\mathrm{c}} \sim 4$. In doing so, we derived the length (B4-R4) and width (B4-B3) of the flow from the $5 \sigma$ level contour of the EHV integrated intensity, and assumed that this comes from only one molecular outflow (see Sect. 4 for a discussion on the multiplicity of flows in the region). Similarly, the opening angle of the outflow can be estimated from the length of the deconvolved major axis $a$ and the length of the deconvolved minor axis $b$, $\delta=2 \times \arctan (b / a)$. The derived value is $\delta \sim 11^{\circ}$. Alternatively, if the $\mathrm{H}_{2}$ emission in the south of $\mathrm{R} 4$ is associated with the same outflow, the collimation factor would be $\sim 6$ and the opening angle $8^{\circ}$. Assuming a distance of $1 \mathrm{kpc}$, the projected extension of the outflow on the sky is $0.2-0.3 \mathrm{pc}$.

\section{2. $\mathrm{CO}$ outflow vs. $\mathrm{H}_{2}$ emission}

Figure 3 compares the red- and blue-shifted CO emission with the $\mathrm{H}_{2}$ at $2.12 \mu \mathrm{m}$ map and the $4.5 \mu \mathrm{m}$ continuum emission from the Spitzer Space Observatory (Benjamin et al. 2003). This emission is often attributed to $\mathrm{H}_{2}$ lines tracing shocked gas arising from outflow activity (Noriega-Crespo et al. 2004; Ybarra \& Lada 2009), although contamination from rovibrational lines of $\mathrm{CO}$ may also happen in hot gas (Marston et al. 2004). $\mathrm{H}_{2}$ emission at $2.12 \mu \mathrm{m}$ is found in correspondence to all the blue-shifted clumps. Emission at $4.5 \mu \mathrm{m}$ is detected towards both B3 and B4. Interestingly, the EHV B3 and B4 clumps are located at the edges of the IR bow-structure traced by $\mathrm{H}_{2}$ and by the $4.5 \mu \mathrm{m}$ emission, and could trace material recently shocked.

Probably due to extinction, only R1 among the red-shifted clumps shows an $\mathrm{H}_{2}$ counterpart (see discussion in paragraph Sect. 4). Figure 3 shows also $\mathrm{H}_{2}$ emission $\sim 40^{\prime \prime}$ south of the YSOs, but it is not clear whether it is tracing emission related to the red-shifted IRAS 17233 outflow. The EHV R1 clump lies ahead of an independent, short $\mathrm{H}_{2}$ jet (see Fig. 6): also in this case it seems we are observing material accelerated along the jet traced by $\mathrm{H}_{2}$ (see Sect. 4).

\subsection{Emission from other molecular species}

Together with the main isotopologue of $\mathrm{CO}$, also the ${ }^{13} \mathrm{CO}$, $\mathrm{C}^{18} \mathrm{O}(2-1)$, and $\mathrm{SO}\left(5_{6}-4_{5}\right)$ transitions show non-Gaussian wings in the spectrum taken at the position of the mm continuum emission (Fig. 2). Figure 7 shows the integrated intensity of the three transitions in the red-and blue-shifted wings. For the red-shifted emission ${ }^{13} \mathrm{CO}$ emission, we only used the range of velocities $(15,20) \mathrm{km} \mathrm{s}^{-1}$ because of contamination from other molecular species.

The emission traced by the three molecular species is more compact than that traced by ${ }^{12} \mathrm{CO}$, as expected. For the redshifted emission, the ${ }^{13} \mathrm{CO}$ emission extends towards the features $\mathrm{R} 2$ and $\mathrm{R} 3$, while the $\mathrm{SO}\left(5_{6}-4_{5}\right)$ transitions has a $5 \sigma$ detection on $\mathrm{R} 2$ and extends towards the $\mathrm{R} 1$ position, where EHV red-shifted $\mathrm{CO}(2-1)$ and $\mathrm{H}_{2}$ are detected. For the blueshifted emission, all transitions are detected on B1, although none of them peaks at this position. All three transitions show two spatially separated blue-shifted emission peaks; the same behaviour is seen in the $\mathrm{CO}(2-1)$ line at velocities between -40 and $-20 \mathrm{~km} \mathrm{~s}^{-1}$, similar to the range of velocities used to obtain the blue-shifted integrated maps of ${ }^{13} \mathrm{CO}$ and SO. However, the position of the two peaks is not the same for the four species. The east blue-shifted peak is probably associated with an outflow traced by a $\mathrm{H}_{2}$ jet and by the $\mathrm{H}_{2} \mathrm{O}$ masers (see discussion in the following paragraph); the west peak is probably related to one of the other two outflows of the region. 


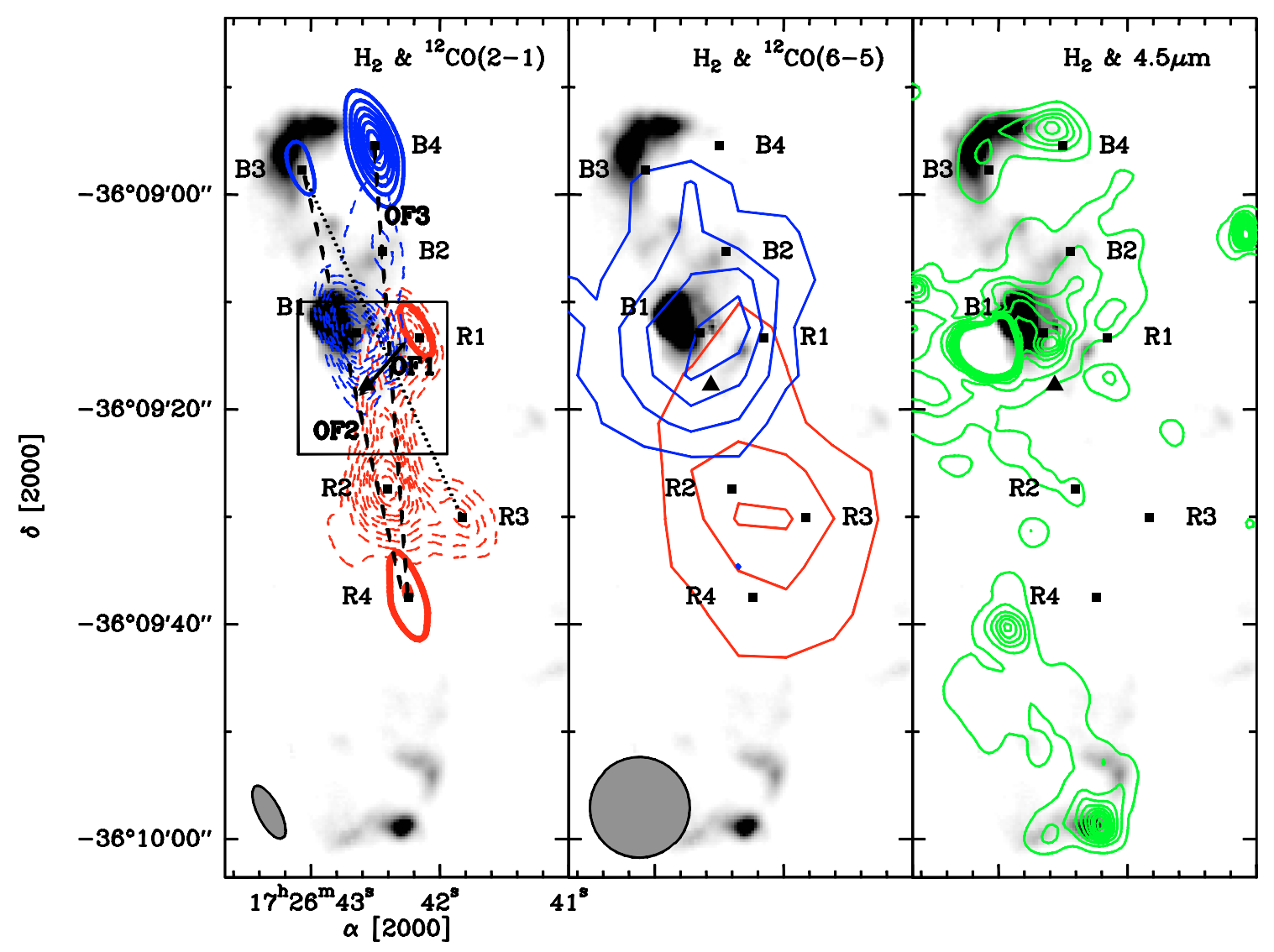

Fig. 3. Integrated emission of the blue- and red-shifted wings in the $\mathrm{CO}(2-1)$, and (6-5) lines, and continuum emission at $4.5 \mu \mathrm{m}$ overlaid on the $\mathrm{H}_{2}$ emission (grey scale). For the $\mathrm{CO}(2-1)$ transition, the solid contours show the EHV blue- $\left(v=[-200,-130] \mathrm{km} \mathrm{s}^{-1}\right)$ and red-shifted emission $\left(v=[90,120] \mathrm{km} \mathrm{s}^{-1}\right)$; the dashed contours mark the HV blue- $\left(v=[-130,-25] \mathrm{km} \mathrm{s}^{-1}\right)$ and red-shifted emission $\left(v=[16,50] \mathrm{km} \mathrm{s}^{-1}\right)$. For the $\mathrm{CO}(6-5)$ line, the solid contours show the blue- $\left(v=[-80,-15] \mathrm{km} \mathrm{s}^{-1}\right)$ and red-shifted emission $\left(v=[8,60] \mathrm{km} \mathrm{s}^{-1}\right)$. All contours start from a $5 \sigma$ level and are in step of $5 \sigma$ (see Table 2 for the rms noises). The squares mark the positions of the red-shifted, blue-shifted CO(2-1) peaks, the triangle the $\mathrm{CH}_{3} \mathrm{OH}$ masers. The beam of each observational dataset is shown in the bottom left corner. In the left panel, the dashed lines and the solid arrow outline the possible molecular outflows discussed in Sect. 4 and labelled IRAS 17233-OF1, -OF2, -OF3. The dotted line marks the possible connection between R3 and B3. The solid lines in the left panel outline the region mapped in Fig. 6.

Table 2. Noise level in the integrated intensity maps presented in Figs. 3 and 7.

\begin{tabular}{lcr}
\hline \hline & Transition & $\begin{array}{c}\text { rms } \\
{\left[\text { Jy beam }^{-1}\right]}\end{array}$ \\
\hline $\mathrm{CO}(2-1)$ & red-shift., HV & 0.5 \\
$\mathrm{CO}(2-1)$ & red-shift., EHV & 1.3 \\
$\mathrm{CO}(2-1)$ & blue-shift., HV & 8.0 \\
$\mathrm{CO}(2-1)$ & blue-shift., EHV & 1.3 \\
$\mathrm{CO}(6-5)$ & red-shift. & 1035 \\
$\mathrm{CO}(6-5)$ & blue-shift. & 863 \\
${ }^{13} \mathrm{CO}(2-1)$ & red-shift. & 0.3 \\
${ }^{13} \mathrm{CO}(2-1)$ & blue-shift. & 1.0 \\
$\mathrm{C}^{18} \mathrm{O}(2-1)$ & red-shift. & 0.4 \\
$\mathrm{C}^{18} \mathrm{O}(2-1)$ & blue-shift. & 0.7 \\
$\mathrm{SO}\left(5_{6}-45\right)$ & red-shift. & 0.4 \\
$\mathrm{SO}\left(5_{6}-4_{5}\right)$ & blue-shift. & 2.0 \\
\hline
\end{tabular}

\section{Multiple molecular outflows from IRAS 17233}

A zoom in the central region of the molecular outflow reveals a complex picture. Figure 6 shows the continuum emission at $1.3 \mathrm{~mm}$ towards the inner region of the outflow detected in $\mathrm{CO}$; overlaid on the map are the $1.3 \mathrm{~cm}$ continuum emission from Zapata et al. (2008), the positions of the $\mathrm{OH}, \mathrm{H}_{2} \mathrm{O}$ and $\mathrm{CH}_{3} \mathrm{OH}$ maser spots (Fish et al. 2005; Zapata et al. 2008; Walsh et al. 1998), the $\mathrm{CO}(2-1) \mathrm{HV}$ blue-shifted emission, the SO blue- and red-shifted emission, and the $\mathrm{H}_{2}$ emission.

Four compact sources are detected in cm continuum emission within $\sim 2^{\prime \prime}$ from the peak of the mm continuum emission (and labelled VLA 2a, 2b, 2c and 2d by Zapata et al. 2008). The brightest source, VLA $2 \mathrm{a}$, is at 0.7 from the mm continuum peak, suggesting that the two sources probably coincide. The $\mathrm{cm}$ VLA 2a emission reveals two components, one dominated by a classical HII region, the other likely due to dust emission. On the other hand, VLA 2 b shows a negative spectral index, possibly associated with synchrotron emission. Finally, VLA 2c and $2 \mathrm{~d}$ have a spectral energy distribution compatible with a hypercompact HII region (HCHII) or with a massive dusty core or disk.

Other information on the sources comes from the maser emission. The $\mathrm{CH}_{3} \mathrm{OH}$ masers (black triangles in Fig. 6), so far detected only towards massive stars (e.g., Xu et al. 2008), seem to indicate their association with VLA $2 \mathrm{~b}$, although with an uncertainty of $\sim 1^{\prime \prime}$ in the absolute position. A bipolar outflow is detected in $\mathrm{OH}$, with blue-shifted emission towards the north and red-shifted emission towards the south as for the $\mathrm{CO}$ emission. $\mathrm{OH}$ masers (filled squares) are observed from molecular material surrounding ultra-compact HII regions, and from protostellar outflows associated or not with cm continuum (e.g., Argon et al. 2003). In the case of $\mathrm{OH}$ masers tracing molecular outflows, the 
B4

B3

B2

B1

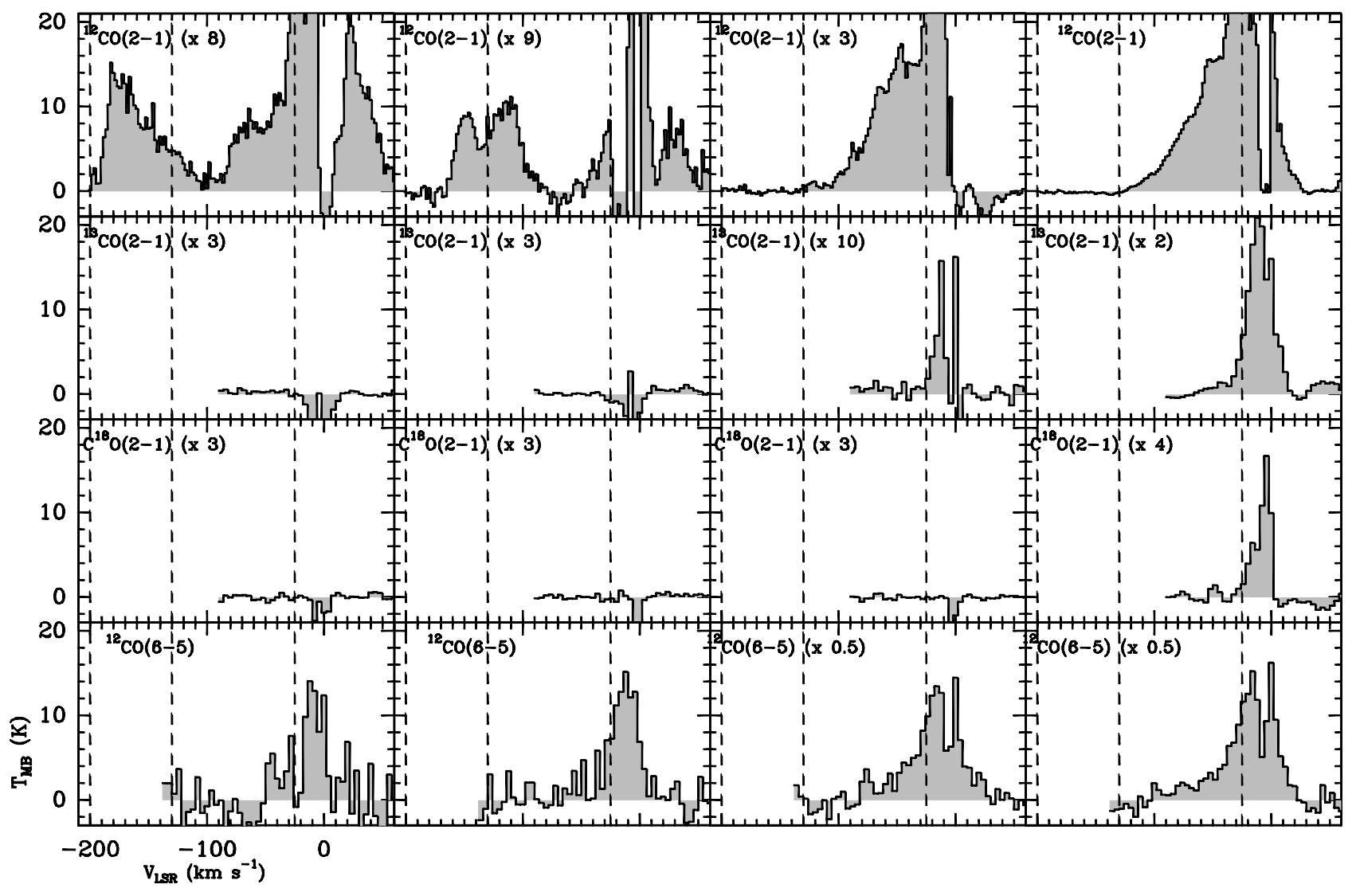

Fig. 4. Spectra of $\mathrm{CO}$ and its isotopologues at the peaks of the $\mathrm{CO}(2-1)$ blue-shifted emission. The dashed lines mark the velocities $-200,-130$ and $-25 \mathrm{~km} \mathrm{~s}^{-1}$ used to define the EHV and HV regimes. Absorption features are probably due to filtering of large structures.

non detection of an ultra-compact HII region can be explained with a very early evolutionary stage of the source, or with an intermediate mass object which will never produce a detectable ultra-compact HII region. In our case, VLA $2 \mathrm{~d}$ could be the driving source of this outflow. Finally, we have a pattern formed by red- and blue-shifted $\mathrm{H}_{2} \mathrm{O}$ masers (empty triangles), which are well known tracers of molecular jets/outflows, spread around the $1.3 \mathrm{~mm}$ continuum peak.

Given the current resolution of our $\mathrm{CO}$ observations and the multiplicity of the high-velocity clumps, we cannot derive the exact number of molecular outflows in the region, in particular those extended along the $\mathrm{N}-\mathrm{S}$ direction. However, using the high angular resolution $\mathrm{H}_{2}$ and maser emission data, we can try to draw a scenario where at least three molecular outflows originate from the maser zone in IRAS 17233:

1. the first one (IRAS 17233-OF1, indicated by a solid arrow in Figs. 3 and 6) is well traced by a $\mathrm{H}_{2}$ jet which seems to arise from the $1.3 \mathrm{~mm}$ continuum emission peak: the driving source could be either VLA $2 \mathrm{a}$ or VLA $2 \mathrm{~b}$. In addition, the $\mathrm{H}_{2} \mathrm{O}$ spots are well in agreement with a molecular outflow directed along the NW(red)-SE(blue) direction. The present ${ }^{12} \mathrm{CO}$ maps confirm this possibility, with high-velocity blue emission located SE of the $1.3 \mathrm{~mm}$ peak (Fig. 6), and the R1 red clump which is located ahead the $\mathrm{H}_{2} \mathrm{NW}$ jet. This picture is consistent with the scenario depicted by SO, a wellknown molecule whose abundance is enhanced in molecular outflows (e.g., Bachiller \& Perez Gutierrez 1997; Codella et al. 2005): blue-shifted emission east of the $1.3 \mathrm{~mm}$ peak, and red-shifted emission in the NW (Fig. 6). Blue-shifted emission is also detected in ${ }^{13} \mathrm{CO}$ and $\mathrm{C}^{18} \mathrm{O}$ (Fig. 7) in the $\mathrm{SE}$ of the $1.3 \mathrm{~mm}$ peak. Thus, the R1 clump seems to trace EHV gas associated with the mass loss traced by $\mathrm{H}_{2}$. The scenario for the SE blue lobe is less clear: we have high velocity blue emission but no EHV clump and, surprisingly, there is no $\mathrm{H}_{2}$ counterpart, when usually the blue $\mathrm{H}_{2}$ emission is brighter than the red one due to extinction. A possible explanation would be that the blue-shifted jet is leaving the high density clump hosting the YSOs, moving in a gas where the density is so low that $\mathrm{H}_{2}$ is not excited. A more realistic solution is that the driving source of the $\mathrm{H}_{2} \mathrm{NW}$ jet (VLA 2a or VLA 2b) had formed inside the $1.3 \mathrm{mmm}$ core, but close to its far edge; in this case the red-shifted jet would travel in a less dense ambient medium, while the blue-shifted jet would travel deeply into the molecular cloud with a consequently higher extinction;

2. a second outflow can be found along the direction traced by the B3-B1-R2-R4 clumps (IRAS 17233-OF2, dashed line in Figs. 3 and 6). Note that this direction is well aligned with both the $\mathrm{OH}$ bipolar outflow and definitely supports VLA 2d as driving source;

3 . the third one can be found along the path traced by the B4B2-R2-R4 clumps (IRAS 17233-OF3). In this case, the corresponding dashed line of Figs. 3 and 6 passes through the $\mathrm{H}_{2} \mathrm{O}$ masers and close to the VLA $2 \mathrm{a} / 1.3 \mathrm{~mm}$ source, which could then host the driving source.

This picture has to be considered as a first incomplete step to prepare higher angular resolution observations, in CO or in a classical tracer of the hot jet component as $\mathrm{SiO}$, which are needed for 
R4 R3

R2

R1

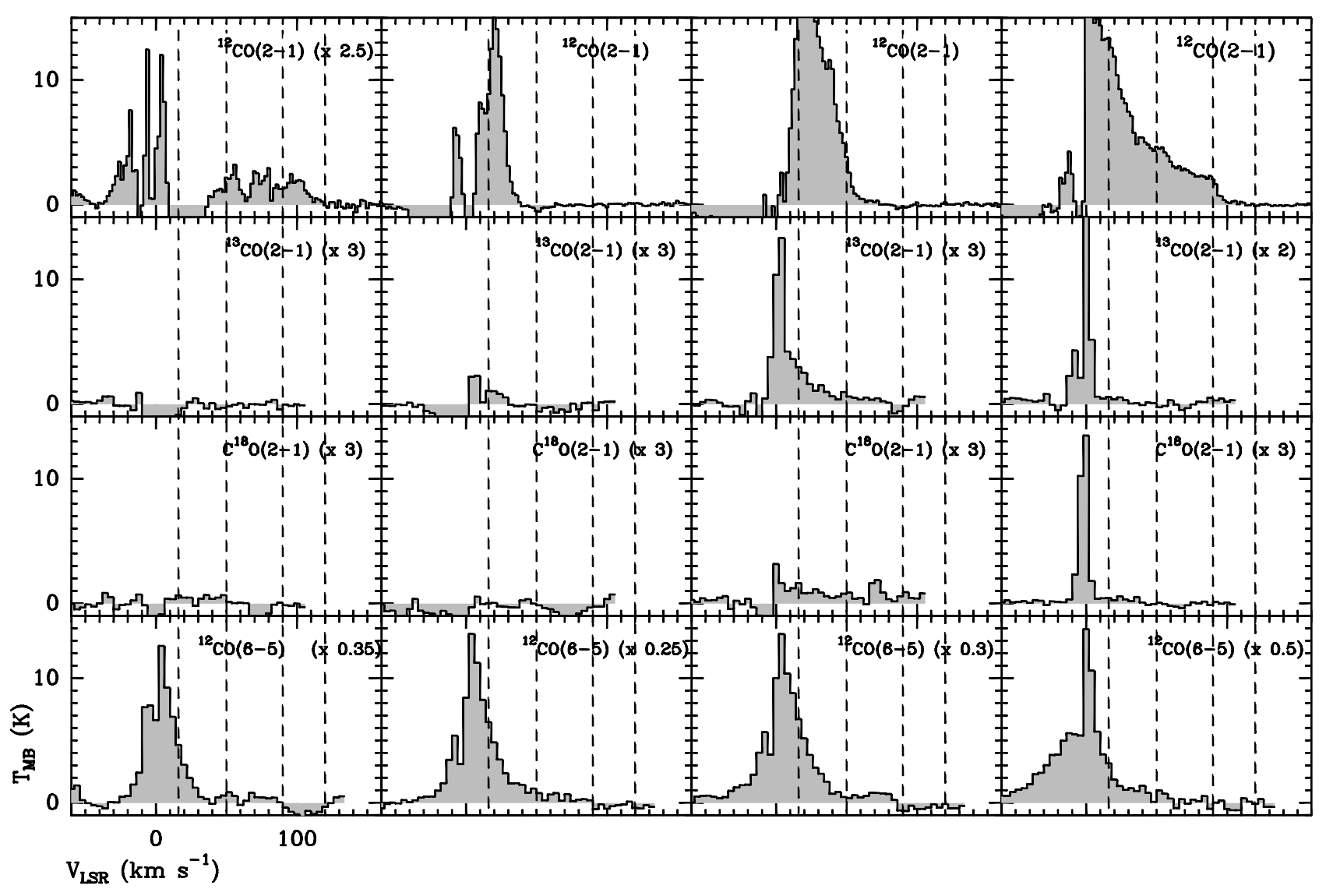

Fig. 5. Spectra of $\mathrm{CO}$ and its isotopologues at the peaks of the $\mathrm{CO}(2-1)$ red-shifted emission. The dashed lines mark the velocities $120,90,50$ and $16 \mathrm{~km} \mathrm{~s}^{-1}$ used to define the EHV and HV regimes. Absorption features are probably due to filtering of large structures.

a better understanding of the number of outflows in the region, and for the identification of the powering sources. For instance, there is no clear solution accounting for the R3 clump. A possible blue counterpart of R3 could be found in B3 and the driving source should be consequently in the $1.3 \mathrm{~mm}$ object. Similarly, $\mathrm{R} 2$ and R4 are not clearly assigned to any given outflows, but in our current picture they could either belong to IRAS 17233-OF2 or to IRAS 17233-OF3.

In conclusion, although higher angular resolution data are needed for a better understanding of the number of outflows in the region, and for the identification of the powering sources, on the basis of the existing data we can safely conclude that at least three molecular outflows are found in the maser zone in IRAS 17233. Given their association with $\mathrm{CH}_{3} \mathrm{OH}$ and $\mathrm{OH}$ maser emission, and given the presence of at least four compact sources of cm continuum emission, we conclude that the molecular outflows originate from intermediate or high-mass YSOs.

\section{Derived outflow parameters}

Global properties of outflows can be derived, under given assumptions, from the high-velocity $\mathrm{CO}$ emission. We will use the ${ }^{13} \mathrm{CO}(2-1),{ }^{12} \mathrm{CO}(2-1)$ and ${ }^{12} \mathrm{CO}(6-5)$ data to infer the optical depth and the excitation temperature. However, there is a major caveat in our analysis: interferometric data are affected by missing flux from extended structures. Moreover, optically thick lines have a more uniform distribution than optically thin transitions, and may be more sensitive to interferometric spatial filtering. Thus, in the following discussion we may (1) overestimate the optical depth of the ${ }^{13} \mathrm{CO}(2-1)$ line; (2) overestimate the ratio between the ${ }^{12} \mathrm{CO}(6-5)$ and the ${ }^{12} \mathrm{CO}(2-1)$ lines, and therefore (3) overestimate the excitation temperature of the gas. In both cases, the problem comes from the ${ }^{12} \mathrm{CO}(2-1)$ transition, whose observed flux may be a lower limit to its true value. Since no single dish ${ }^{12} \mathrm{CO}(2-1)$ observations of IRAS 17233 are available, we cannot further investigate this possibility. In Sect. 5.1, we will derive the optical depth of the ${ }^{13} \mathrm{CO}(2-1)$ and ${ }^{12} \mathrm{CO}(2-1)$ lines; in Sect. 5.2, we will investigate the excitation conditions of the gas along the outflow. Finally, in Sect. 5.3 we will derive the properties of the outflow making use of the opacities derived in Sect. 5.1 and for a range of temperatures compatible with the results of the statistical-equilibrium calculations performed in Sect. 5.2.

\subsection{Optical depth}

By comparing the intensity of the ${ }^{13} \mathrm{CO}$ and ${ }^{12} \mathrm{CO}(2-1)$ intensities in the wings, it is possible to estimate the optical depths of both lines, assuming a $\left[{ }^{12} \mathrm{CO} /{ }^{13} \mathrm{CO}\right]$ relative abundance of 77 (Wilson \& Rood 1994). We estimated the optical depth of the ${ }^{13} \mathrm{CO}(2-1)$ line, $\tau_{13}$, under the assumptions that the ${ }^{13} \mathrm{CO}(2-1)$ and ${ }^{12} \mathrm{CO}(2-1)$ lines have the same excitation temperature, and that the ${ }^{12} \mathrm{CO}(2-1)$ transition is optically thick. Since (i) the $\mathrm{S} / \mathrm{N}$ ratio in the ${ }^{13} \mathrm{CO}(2-1)$ wings is sufficiently high only in the relatively low velocity outflow range; and (ii) self-absorption affects the ${ }^{12} \mathrm{CO}(2-1)$ line at velocities close to the ambient one, we derived the optical depths in the $-55,-20 \mathrm{~km} \mathrm{~s}^{-1}$ (red) and $+15,+25 \mathrm{~km} \mathrm{~s}^{-1}$ (blue) ranges. Figure 8 shows the distribution of the optical depth of the ${ }^{13} \mathrm{CO}(2-1)$ line averaged over these 


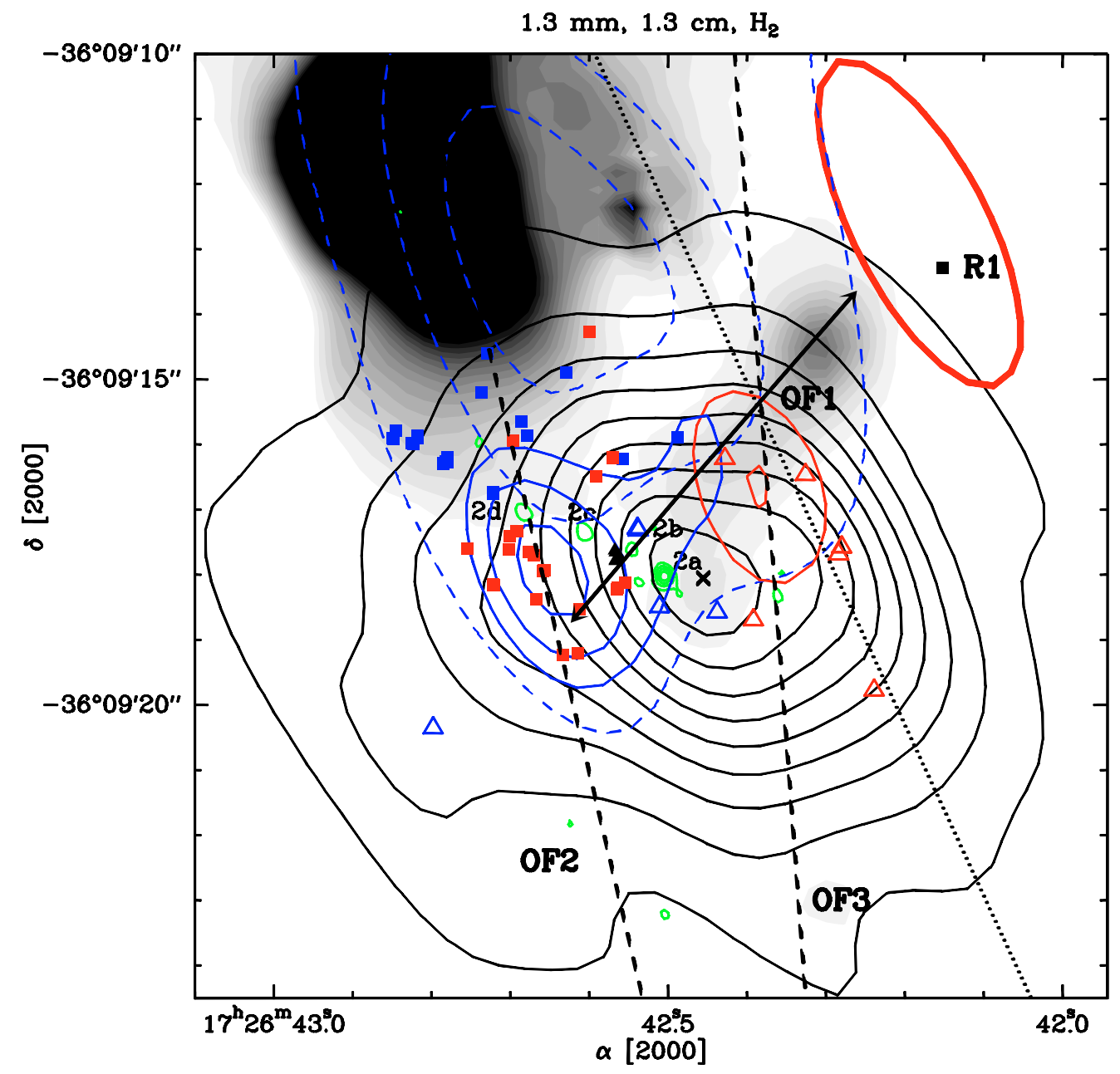

Fig. 6. Continuum emission at $1.3 \mathrm{~mm}$ observed with the SMA (Leurini et al., in prep.) from the maser zone in IRAS 17233 (solid black contours from $0.12 \mathrm{Jy} \mathrm{beam}^{-1}(3 \sigma)$ in step of $0.24 \mathrm{Jy}_{\text {beam }}{ }^{-1}$ ). Overlaid on the map are the positions of the $\mathrm{OH}$ (blue and red squares, Fish et al. 2005), of the $\mathrm{CH}_{3} \mathrm{OH}$ (black triangles, Walsh et al. 1998) and of the water (blue and red open triangles, Zapata et al. 2008) masers. The green contour levels show the continuum emission at $1.3 \mathrm{~cm}$ (from $1.4 \mathrm{mJy} \mathrm{beam}^{-1}(3 \sigma)$ in step of $1.4 \mathrm{mJy}$ beam $^{-1}$, Zapata et al. 2008), while the grey scale map is the $\mathrm{H}_{2}$ emission. The dashed blue contour levels represent the $\mathrm{CO}(2-1)$ blue-shifted emission at high velocities (from $5 \sigma$ in step of $20 \sigma$, see Table 2 ), the solid blue and red contours the $\mathrm{SO}\left(5_{6}-4_{5}\right)$ blue- and red-shifted emission (blue: from 80 to $104 \mathrm{Jy} \mathrm{beam}^{-1}$ in step of $10 \mathrm{Jy} \mathrm{beam}^{-1}$; red: 10 and $12 \mathrm{Jy}_{\text {beam }}{ }^{-1}$ ). The black square marks the R1 position, detected in the EHV red-shifted CO(2-1) emission (thik solid red contour, as in Fig. 3). The cross marks the position of the peak position of the $1.3 \mathrm{~mm}$ continuum emission. The dashed and dotted lines and the arrow are as in Fig. 3. $\mathrm{OF} 1, \mathrm{OF} 2$, and OF3 label the three identified outflows.

velocity ranges: the emission in the ${ }^{13} \mathrm{CO}(2-1)$ line results to be optically thin, with $\tau_{13} \leq 0.1-0.3$. The ${ }^{12} \mathrm{CO}(2-1)$ optical depth, $\tau_{12}$, was derived by simply scaling the $\tau_{13}$ value by the assumed $\left[{ }^{12} \mathrm{CO} /{ }^{13} \mathrm{CO}\right]$ ratio, thus resulting in an optical depth for the ${ }^{12} \mathrm{CO}(2-1)$ line, $\tau_{12}$, between 10 and 30 .

\subsection{LVG analysis}

In order to constrain the physical conditions associated with the $\mathrm{CO}$ outflow(s) by means of statistical-equilibrium calculations, we compared the ${ }^{12} \mathrm{CO} J=6-5$ and $2-1$ lines $\left(E_{\mathrm{u}}=116.2\right.$ and $16.6 \mathrm{~K}$, respectively). For a proper comparison, the ${ }^{12} \mathrm{CO}(2-1)$ image, originally obtained with a $5 . \prime 5 \times 2$.' 1 resolution, was reconstructed at the lower resolution of the ${ }^{12} \mathrm{CO}(6-5)$ map $(H P B W=9$ '. 4$)$. We then measured the ratio $R_{62}$ between the brightness temperature of the $J=6-5$ and 2-1 lines. Figure 9 shows the distribution of $R_{62}$ with velocity with respect to the systemic one $\left(V_{\mathrm{LSR}}\right)$ as observed towards the blue- (upper panel) and red-shifted (lower panel) outflow lobes. Interestingly, Fig. 9 shows that, for each blue- and red-shifted lobe the higher the distance of the clump from the driving source(s) is, the higher is the $R_{62}$ ratio, thus indicating higher excitation conditions. For instance, the $R_{62}$ ratio calculated for the $\mathrm{B} 4$ clumps is very high: $\sim 10$. However, the $\mathrm{CO}$ emission is associated with multiple outflows, and among the $\mathrm{CO}$ peaks only B2 and B4 clearly appear to be related to the same flow. Therefore, the trend seen in the $R_{62}$ ratio may not be physically relevant for the other positions. Higher angular resolution observations are needed to get a clearer picture of the region. In addition, Fig. 9 also suggests, at least for the red clumps, an increase of the excitation at the highest velocities, probably closely associated with recently shocked gas.

In order to constrain the excitation conditions, we used a Large Velocity Gradient (LVG) code with collisional rates from Schöier et al. (2005). These include extrapolation to energy levels up to $J=40$ and collisional temperatures up to $2000 \mathrm{~K}$, based on the original datasets calculated by Flower (2001) and Wernli et al. (2006) for temperatures in the range from 5 to $400 \mathrm{~K}$ and energy levels up to $J=29$ and $J=20$ for collisions with para$\mathrm{H} 2$ and ortho-H2, respectively. 


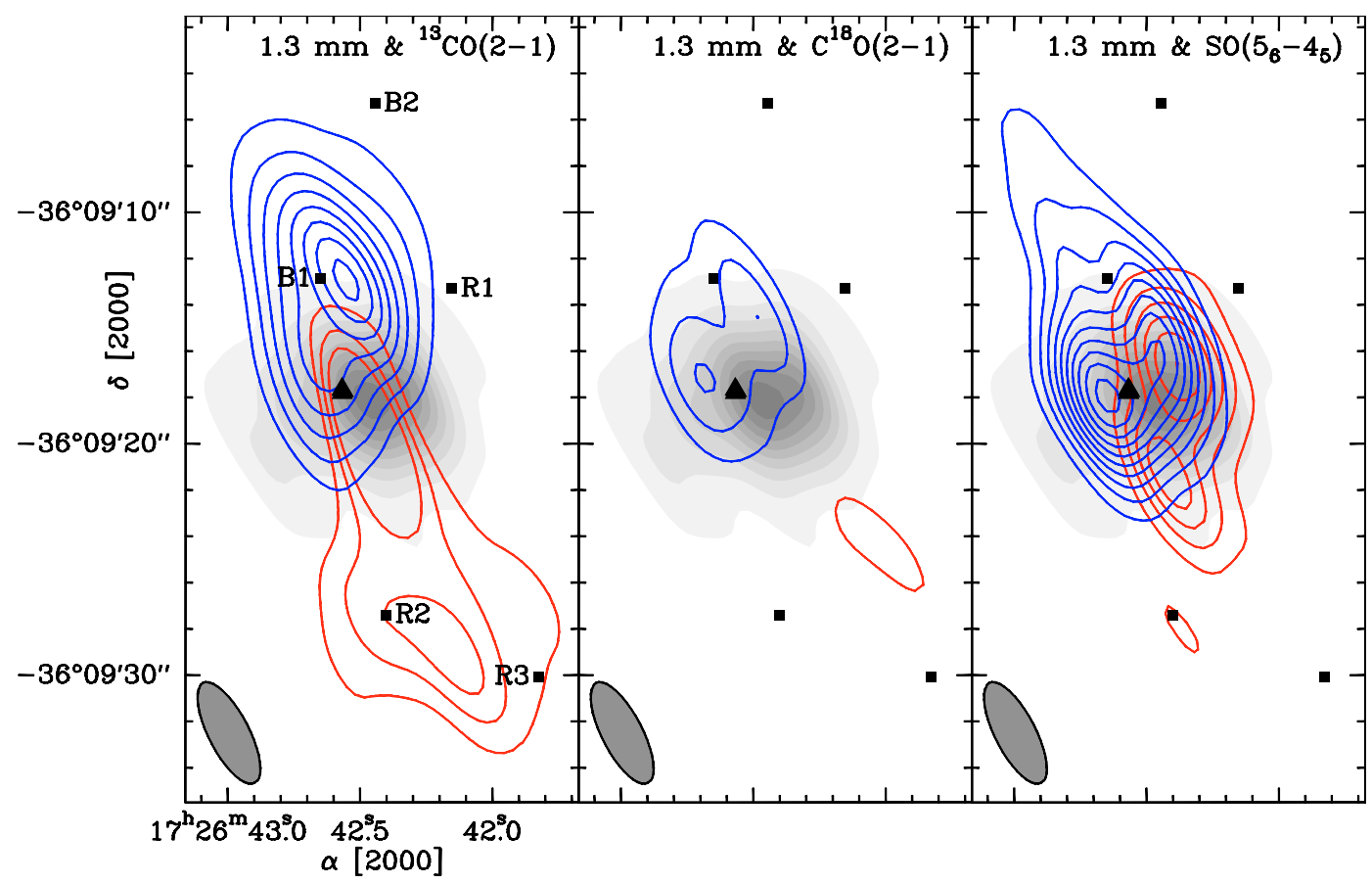

Fig. 7. Maps of the integrated blue- and red-shifted emission in the ${ }^{13} \mathrm{CO}(2-1), \mathrm{C}^{18} \mathrm{O}(2-1)$ and $\mathrm{SO}\left(5_{6}-4_{5}\right)$ transitions. The solid contours show the blue-shifted emission in the velocity ranges $v=[-55,-20] \mathrm{km} \mathrm{s}^{-1}$ for the ${ }^{13} \mathrm{CO}(2-1)$ line, $v=[-20,-11] \mathrm{km} \mathrm{s}^{-1}$ for $\mathrm{C}^{18} \mathrm{O}(2-1)$ and $v=$ $[-40,-11] \mathrm{km} \mathrm{s}^{-1}$ for $\mathrm{SO}\left(5_{6}-45\right)$, and the red-shifted emission in the velocity range $v=[15,20] \mathrm{km} \mathrm{s}^{-1}$ for the ${ }^{13} \mathrm{CO}(2-1)$ line, $v=[5,10] \mathrm{km} \mathrm{s}{ }^{-1}$ for $\mathrm{C}^{18} \mathrm{O}(2-1)$ and $v=[15,25] \mathrm{km} \mathrm{s}^{-1}$ for $\mathrm{SO}\left(5_{6}-45\right)$. The contours start from a $5 \sigma$ level and are in step of $5 \sigma$. The grey scale map is the continuum emission at $1.3 \mathrm{~mm}$ (from $0.12 \mathrm{Jy}_{\text {beam }}^{-1}(3 \sigma)$ in step of $0.24 \mathrm{Jy}_{\text {beam }}{ }^{-1}$ ). The symbols are as in Fig. 3. The synthesised beam is shown in the bottom left corner.

We assumed a specific column density $N_{\mathrm{CO}} \Delta v^{-1}$ ranging from $10^{14}$ to $10^{19} \mathrm{~cm}^{-2}\left(\mathrm{~km} \mathrm{~s}^{-1}\right)^{-1}$. Figure 10 shows our typical model results, illustrated graphically with the ratio $R_{62}$ (grey scale) as a function of the thermal pressure $\left(T_{\mathrm{kin}} \times n_{\mathrm{H}_{2}}\right)$ and the kinematic temperature $\left(T_{\text {kin }}\right)$. Figure 10 shows the solutions found for $N_{\mathrm{CO}} \Delta v^{-1}=10^{16} \mathrm{~cm}^{-2}\left(\mathrm{~km} \mathrm{~s}^{-1}\right)^{-1}$ : however, this should be considered as an upper limit since also solutions down to $10^{14} \mathrm{~cm}^{-2}\left(\mathrm{~km} \mathrm{~s}^{-1}\right)^{-1}$ are acceptable. Solid lines mark the measured values of $R_{62}$ towards the red- (upper panel) and blue-shifted (lower panel) outflow lobes. For clarity, we plotted the values averaged over the whole velocity ranges. In addition, we further averaged the measurements of the clumps associated with the lower excitation conditions, showing similar values (red: $R_{62}(\mathrm{R} 1, \mathrm{R} 2)=\left(R_{62}(\mathrm{R} 1)+R_{62}(\mathrm{R} 2)\right) / 2$; blue: $R_{62}(\mathrm{~B} 1, \mathrm{~B} 2)=\left(R_{62}(\mathrm{~B} 1)+R_{62}(\mathrm{~B} 2)\right) / 2$, where $R_{62}$ at each position is the average over the values for different velocities). Dot-dashed lines are for the $R_{62}$ uncertainties, defined as the minimum and maximum values that $R_{62}(\mathrm{R} 1, \mathrm{R} 2)$ reaches.

Figure 10 clearly shows that we cannot obtain severe constraints on the physical conditions of the gas with only two transitions. However, it allows us to quantify the different excitation conditions of the outflow clumps. Regarding the red lobe, the R1 and R2 clumps have $T_{\text {kin }} \geq 50 \mathrm{~K}$ and a product $T_{\text {kin }} \times n_{\mathrm{H}_{2}}$, which can be considered a measure of the gas pressure, $\geq 10^{6} \mathrm{~K} \mathrm{~cm}^{-3}$. A similar scenario can be found in the blue lobes B1 and B2. On the other hand, the $R_{62}$ value towards the extreme high velocity position B4 is out of the plane sampled by our models $\left(R_{62} \sim 10\right)$. The red-shifted extreme high velocity position $\left(\mathrm{R} 3, R_{62} \sim 7\right)$ is at the limit of the plane. Such high values of $R_{62}$ suggest very high temperatures ( $\geq 150 \mathrm{~K}$ for R3 and $\geq 300 \mathrm{~K}$ for B4) for the gas at those positions; however, these values must be taken as upper limits since the flux of the $\mathrm{CO}(2-1)$ line could be underestimated due to filtering of large structures, as stated in Sect. 5.

\subsection{Outflow energetics and kinematics}

The outflow mass, moment and energy ( $M_{\text {out }}, p_{\text {out }}$, and $E_{\text {out }}$, respectively) were derived from line emission using the classical formulae (e.g., Lada 1985) as a sum over all the velocity channels within the blue and red wings and within the areas defined by the $3 \sigma$ contour levels of the respective outflow lobes. We sum all the individual pixel contributions within the lobe area. In other words, the sum of the contributions from each pixel and velocity channel builds up the total outflow mass, moment and energy. We used the ${ }^{12} \mathrm{CO}(2-1),{ }^{12} \mathrm{CO}(6-5)$, and ${ }^{13} \mathrm{CO}(2-1)$ lines separately in order to have different estimates. The abundance ratio $\left[{ }^{12} \mathrm{CO}\right] /\left[\mathrm{H}_{2}\right]$ and $\left[{ }^{13} \mathrm{CO}\right] /\left[\mathrm{H}_{2}\right]$ was taken as $10^{-4}$ and $1.3 \times 10^{-6}$ (e.g., Scoville et al. 1986), respectively. We conservatively assumed an excitation temperature $T_{\mathrm{ex}}$ in the range $50-200 \mathrm{~K}$ for all velocities, following the LVG analysis. We corrected the $M_{\text {out }}, p_{\text {out }}$, and $E_{\text {out }}$ for the optical depths derived in Sect. 5.1. The average distance of $1000 \mathrm{pc}$ has been used. The ${ }^{12} \mathrm{CO}$ molecule is the usual outflow tracer due to its high abundance, which allows to detect the weakest wings at the highest velocity. Nevertheless, the risk of ${ }^{12} \mathrm{CO}$ lies in its high opacity, which can bias the observations towards the colder and more extended portion of the outflow. We tried to verify this possibility by using also the ${ }^{12} \mathrm{CO}(6-5)$ and ${ }^{13} \mathrm{CO}(2-1)$ lines, which present weaker emission, thus preventing the detection of the highest velocities, but which could possibly better trace the more collimated outflow component.

Table 3 lists both the values derived summing the contribution due to all the outflows present in the region (hereafter whole emission) as well as the values of the OF1 red-shifted outflow. In the latter case, we refer to the ${ }^{12} \mathrm{CO}(2-1)$ line only, since it is the only transition where the OF1 outflow is clearly detected. Note that, for the whole emission, we do not indicate $\tau_{\text {blue }}$ and $\tau_{\text {red }}$, 


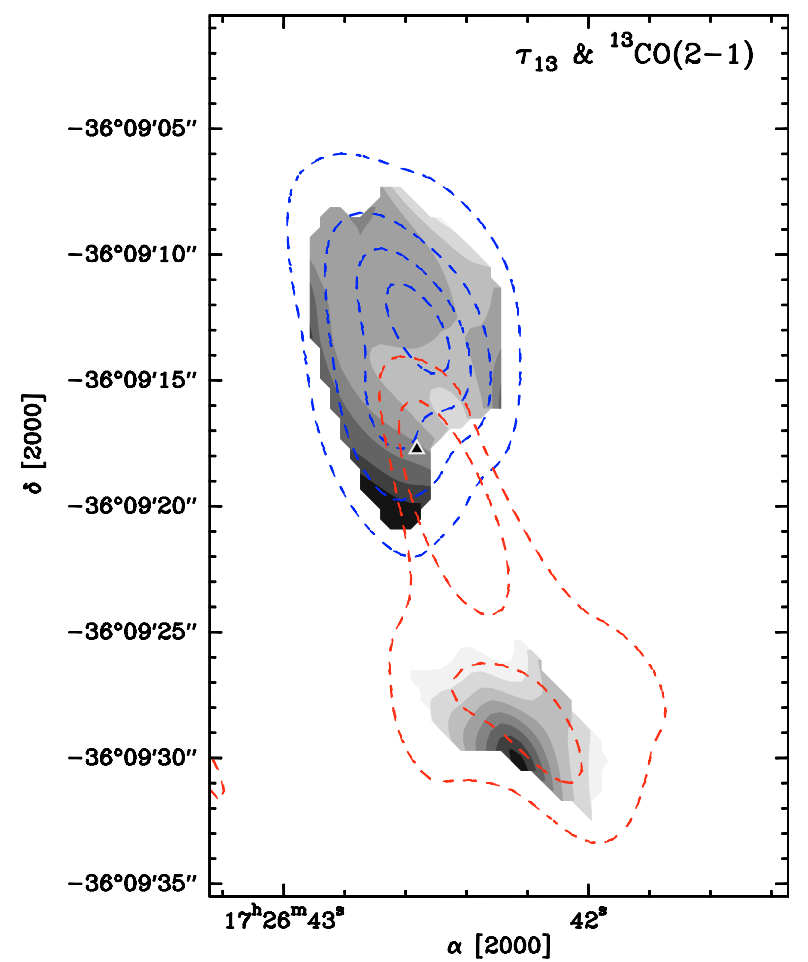

Fig. 8. Overlay of the opacity of the ${ }^{13} \mathrm{CO}$ wings $\left(\tau_{13}\right.$; grey scale, levels are from 0.07 (light grey) to 0.12 (dark grey) in step of 0.01 ) as averaged in the $-55,-20 \mathrm{~km} \mathrm{~s}^{-1}$ (red) and $+15,+25 \mathrm{~km} \mathrm{~s}^{-1}$ (blue) ranges, and the ${ }^{13} \mathrm{CO}$ integrated blue- and red-shifted outflow (see Fig. 7: for clarity, only selected contour levels are drawn). The triangle marks the position of the $\mathrm{CH}_{3} \mathrm{OH}$ methanol masers (see Fig. 6).

but, for clarity, the mean value, $\tau_{\text {mean }}=\left(\tau_{\text {blue }}+\tau_{\text {red }}\right) / 2$, which can differ slightly. However, the average values of the optical depth in the red- and blue-shifted lobes, $\tau_{\text {blue }}$ and $\tau_{\text {red }}$, were used in the calculations. For the ${ }^{12} \mathrm{CO}(2-1)$ line, we adopt $\tau_{\text {blue }}=15$ and $\tau_{\text {red }}=23$; for the ${ }^{13} \mathrm{CO}(2-1)$ transition, $\tau_{\text {blue }}=0.2$ and $\tau_{\text {red }}=0.3$; for the ${ }^{12} \mathrm{CO}(6-5)$ line, we used a typical value inferred by the LVG analysis, $\tau_{\text {blue }}=\tau_{\text {red }}=0.1$

From Table 3 we confirm what was expected, i.e. that the ${ }^{12} \mathrm{CO}(2-1)$ line allows us to trace the most energetic portion of the outflow(s) thanks to the detection of the highest velocities. The ${ }^{12} \mathrm{CO}(6-5)$ and ${ }^{13} \mathrm{CO}(2-1)$ lines report $E_{\text {out }}$ lower by more than one order of magnitude. Note also that ${ }^{13} \mathrm{CO}$ is tracing a mass $\sim 2-3$ times lower than that derived by the ${ }^{12} \mathrm{CO}(2-1)$ transition. This is consistent with the fact that only limited velocity ranges of ${ }^{13} \mathrm{CO}(2-1)$ can be used for the integrated intensity in the red-shifted emission due to contamination from several $\mathrm{HC}$ tracers (see Fig. 2), and with the fact that the ${ }^{13} \mathrm{CO}$ emission is more compact than that of the ${ }^{12} \mathrm{CO}(2-1)$ line. In addition, we cannot exclude that the mass estimate is affected by other uncertainties due to the presence of temperature gradients, with the ${ }^{13} \mathrm{CO}$ tracing warmer inner regions, as well as to the optical depth estimate. Therefore, we derived the outflow kinematics only by using the ${ }^{12} \mathrm{CO}(2-1)$ emission.

In order to derive the real outflow parameters, the angle $\theta$ of inclination to the plane of the sky has to be assumed. Unfortunately, because of the complexity of the region and the outflow multiplicity, we cannot derive a good estimate of $\theta$. However, taking into account the quite elongated structure of the outflow(s) and the very limited overlap between red and blue lobes, it is reasonable to assume an intermediate inclination, in the $30^{\circ}-60^{\circ}$ range. A kinematic age for each flow lobe, $t_{\text {kin }}$,

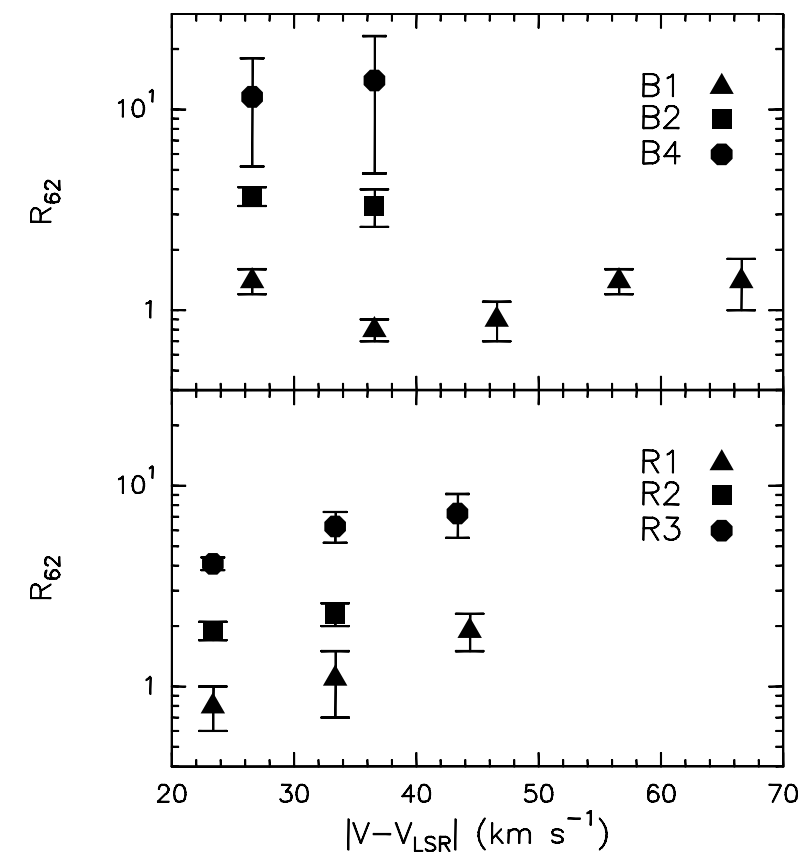

Fig. 9. Distribution with velocity with respect to the systemic one $\left(V_{\mathrm{LSR}}\right)$ of the ratio $R_{62}$ between the brightness temperatures of the $J=6-5$ and 2-1 lines as observed towards the blue- (upper panel) and red-shifted (lower panel) outflow lobes.

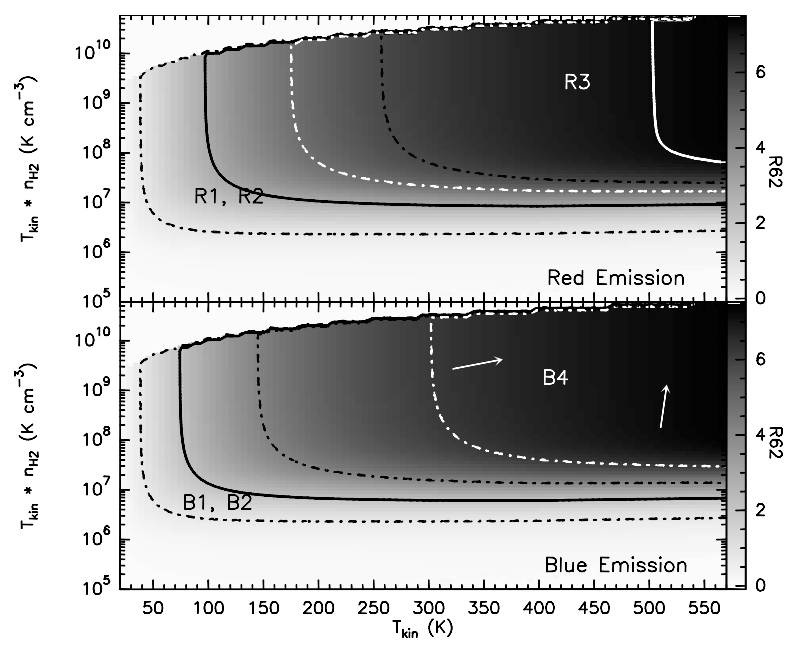

Fig. 10. Results of statistical equilibrium calculations for ${ }^{12} \mathrm{CO}$. The ratio $R_{62}$ (grey scale) between the brightness temperature of the $J=6-5$ and 2-1 lines, as a function of the $T_{\text {kin }} \times n_{\mathrm{H}_{2}}$ product, which can be used as a measure of the gas pressure, and $T_{\text {kin }}$. We have assumed the LVG optical depth parameter $N_{\mathrm{CO}} \Delta v^{-1}=10^{16} \mathrm{~cm}^{-2}\left(\mathrm{~km} \mathrm{~s}^{-1}\right)^{-1}$. Solid lines mark $R_{62}$ as measured towards the red- (upper panel) and blueshifted (lower panel) outflow lobes. Dot-dashed lines are for the $R_{62}$ uncertainties. Note that the $R_{62}$ value towards B4 is out of the sampled plane $(\sim 13)$ : white arrows are drawn to clarify the portion of the plane allowed for B4.

was determined from the projected distance between the position of the outflow lobe and that of the mm-peak, and the corresponding radial outflow velocity component. From the kinematic outflow age, the mass entrainment rate of the molecular outflow, $\dot{M}_{\text {out }}$, the momentum rate, $\dot{p}_{\text {tot }}$, and the mechanical luminosity, $L_{\mathrm{mec}}$, were derived for the blue and red lobes separately, and then added together to obtain the total values presented in Table 4 . There, we list $t_{\text {kin }}, \dot{M}_{\text {out }}, \dot{p}_{\text {tot }}, L_{\text {mec }}$ without correction for the inclination angle, but also for $\theta$ equals $30^{\circ}$ 
Table 3. Whole emission (summing the contribution due to the three identified outflows IRAS 17233-OF1, -OF2 and -OF3) and the IRAS 17233-OF1 outflow energetics.

\begin{tabular}{ccccc}
\hline \hline Tracer & $\tau_{\mathrm{m}}^{\dagger}$ & $\begin{array}{c}M_{\text {out }}{ }^{\ddagger} \\
\left(M_{\odot}\right)\end{array}$ & $\begin{array}{c}p_{\text {out }^{\ddagger}} \\
\left(M_{\odot} \mathrm{km} \mathrm{s}^{-1}\right)\end{array}$ & $\begin{array}{c}E_{\text {out }}{ }^{\ddagger} \\
\left(10^{45} \mathrm{erg}\right)\end{array}$ \\
\hline \multicolumn{5}{c}{ Whole emission (OF1+OF2+OF3) } \\
\hline${ }^{12} \mathrm{CO}(2-1)$ & 19.0 & $2.4-3.7$ & $99-140$ & $60-90$ \\
${ }^{13} \mathrm{CO}(2-1)$ & 0.3 & $1.0-1.5$ & $19-30$ & $4-6$ \\
${ }^{12} \mathrm{CO}(6-5)$ & 0.1 & $0.7-1.1$ & $20-29$ & $6-10$ \\
\hline \multicolumn{5}{c}{ OF1 red-shifted outflow } \\
\hline${ }^{12} \mathrm{CO}(2-1)$ & 23.0 & $0.6-0.9$ & $18-28$ & $6-9$ \\
\hline
\end{tabular}

$\dagger$ Whole emission: mean $\tau$ between blue and red emission optical depths (Sect. 5.1).

$¥$ Values derived for $T_{\text {ex }} 50$ and $200 \mathrm{~K}$ (Sect. 5.2).

and $60^{\circ}$. As done for optical depths, we give a mean value for the kinematic age between the values derived separately for the blue and red emission. After geometry correction, for the whole emission we have a kinematic age of $10^{2}-10^{3} \mathrm{yr}$, and consequently $\dot{M}_{\text {out }} \simeq 2-9 \times 10^{-3} M_{\odot} \mathrm{yr}^{-1}, \dot{p}_{\text {tot }} \simeq 0.1-0.7 M_{\odot} \mathrm{km} \mathrm{s}^{-1} \mathrm{yr}^{-1}$, and $L_{\text {mec }} \simeq 500-8000 L_{\odot}$. By comparing these estimates with the expected correlation between the dynamical parameters and the source bolometric luminosity (e.g., Beuther et al. 2002b), and taking into account the outflow multiplicity, we infer for the driving sources a $L_{\mathrm{bol}} \geq 10^{4} L_{\odot}$, i.e. earlier than a B0.5 type. On the other hand, if we take into account the OF1 outflow only, the bolometric luminosity of the driving source is of the order of $10^{4} L_{\odot}$. The luminosity estimated for the driving sources are well in agreement with the bolometric luminosity of IRAS 17233 , which is $2.5 \times 10^{4} L_{\odot}$ for a distance of $1 \mathrm{kpc}$.

The derived values are in general good agreement with those $\left(M_{\text {out }}=0.8 M_{\odot}, p_{\text {out }}=25.3 M_{\odot} \mathrm{km} \mathrm{s}^{-1}, E_{\text {out }}=8.6 \times 10^{45} \mathrm{erg}\right.$, $t_{\text {kin }}=6200 \mathrm{yr}$ ) reported by Leurini et al. (2008) based on the analysis of single dish data in $\mathrm{CO}(3-2)$, and with the conclusion that the kinematical outflow parameters are typical of massive YSOs. The differences between the two analyses can be explained with the different areas and different velocities adopted for the analysis. Moreover, our previous study on $\mathrm{CO}(3-2)$ did not include any correction for the opacity of the line.

\subsection{Age vs. outflow collimation}

The collimation and the age of the outflows can be compared with the scenario described by Beuther \& Shepherd (2005) where jet-like outflows occur only in early evolutionary phases where no $\mathrm{HCHII}$ region has formed and with stellar luminosities corresponding to late B-type main sequence values, whereas in later evolutionary phases, a HCHII forms and the wind from the central massive star produces an additional less collimated outflow component. Since to form early O-type massive stars via accretion the protostellar objects must accrete even after the central object has reached the main sequence, the evolutionary scenario depicted above also corresponds to a change of luminosity of the (proto)star during its evolution, from a late B-type to an early O-type star before reaching its final mass and stellar luminosity. This scenario would explain the observational result that no collimated jet-like outflow has ever been detected from very young early O-type (proto)stars.

In the IRAS 17233 case, the N-S outflows show a remarkable degree of collimation $\left(f_{\mathrm{c}} \sim 4\right)$ as well as a relatively small opening angle, $\delta \sim 11^{\circ}$. In addition, these estimates have to
Table 4. Whole emission (due to the three identified outflows IRAS 17233-OF1, -OF2 and -OF3) and IRAS 17233-OF1 outflow timescales and kinematics, derived from ${ }^{12} \mathrm{CO}(2-1)$ emission.

\begin{tabular}{ccccc}
\hline \hline $\begin{array}{c}\theta \\
(\mathrm{deg})\end{array}$ & $\begin{array}{c}t_{\text {kin }}{ }^{\dagger} \\
(\mathrm{yr})\end{array}$ & $\begin{array}{c}\dot{M}_{\text {out }}{ }^{\ddagger} \\
\left(M_{\odot} \mathrm{yr}^{-1}\right)\end{array}$ & $\begin{array}{c}\dot{\dot{p}}_{\text {tot }}{ }^{\ddagger} \\
\left(M_{\odot} \mathrm{km} \mathrm{s}^{-1} \mathrm{yr}^{-1}\right)\end{array}$ & $\begin{array}{c}L_{\text {mec }}{ }^{\ddagger} \\
\left(L_{\odot}\right)\end{array}$ \\
\hline \multicolumn{5}{c}{ Whole emission (OF1+OF2+OF3) } \\
\hline 30 & $480-940$ & $3-5 \times 10^{-3}$ & $0.1-0.2$ & $760-1200$ \\
60 & $830-545$ & $5-9 \times 10^{-3}$ & $0.4-0.7$ & $5244-8280$ \\
\hline \multicolumn{5}{c}{ OF1 red-shifted outflow } \\
\hline- & $360-600$ & $1-2 \times 10^{-3}$ & $0.03-0.05$ \\
30 & $209-348$ & $2-3 \times 10^{-3}$ & $0.1-0.2$ & $585-924$ \\
60 & $623-1038$ & $\sim 1 \times 10^{-3}$ & $0.02-0.03$ & $56-132$ \\
\hline
\end{tabular}

$\dagger$ Whole emission: mean $t_{\text {kin }}$ between blue and red emission (Sect. 5.1).

¥ Values derived for $T_{\text {ex }} 50$ and $200 \mathrm{~K}$ (Sect. 5.2).

be considered as lower (collimation) and upper (opening angle) limits, given the unknown outflow multiplicity. The spectral type (B0.5) derived by the outflow parameters and the age of flow $\left(\sim 10^{2}-10^{3} \mathrm{yr}\right)$ agree with the scenario of Beuther \& Shepherd (2005). However, the $\mathrm{OH}$ maser spots trace a bipolar outflow with a definitely larger opening angle (see Fig. 6) than that observed in $\mathrm{CO}$ and $\mathrm{H}_{2}$, thus suggesting we are observing a stage where jet-like and less collimated winds co-exist. The detection of HCHII, typical of more evolved objects, also supports our interpretation that IRAS 17233 is in a transitional phase, when the star also produces an additional less collimated outflow component.

\section{Conclusions}

We presented interferometric (SMA) and single-dish (APEX) observations of one of the nearest massive star forming region IRAS 17233 in $\mathrm{CO}(2-1)$ and (6-5) with angular resolution of 5 .' $4 \times 1$ 1.'9 and 9.'4, respectively. The main results can be summarised as follows:

1. The data reveal a clumpy extended $\left(\sim 50^{\prime \prime}\right)$ structure with well separated blue- and red-shifted emission, and an overall structure roughly aligned along the $\mathrm{N}-\mathrm{S}$ direction and centred on the region where groups of $\mathrm{H}_{2} \mathrm{O}, \mathrm{CH}_{3} \mathrm{OH}$, and $\mathrm{OH}$ maser spots are detected. This region is also associated with the peak of the $1.3 \mathrm{~mm}$ emission we mapped, as well as, on a subarcsec-scale, with 4 HCHII regions (called VLA 2a, b, $\mathrm{c}$ and d) observed at $\mathrm{cm}$-wavelengths and indicating massive star formation. The outflow is associated with extremely high velocities, up to $\sim-200$ and $+120 \mathrm{~km} \mathrm{~s}^{-1}$ with respect to the ambient LSR velocity. Up to eight outflow clumps have been observed in $\mathrm{CO}(2-1)$. The whole outflow structure is very well collimated $\left(f_{\mathrm{c}} \sim 4\right)$ and with an opening angle of $\sim 11^{\circ}$.

2 . The large number of outflow clumps clearly indicates outflow multiplicity, as expected for a cluster of high-mass YSOs. Using high angular resolution maps of $\mathrm{H}_{2}, \mathrm{~cm}-$ continuum, and maser emission, we tried to identify different outflow components. At least three outflows can be distinguished. One outflow (IRAS 17233-OF1) is compact $\left(\sim 5^{\prime \prime}-10^{\prime \prime}\right)$ and located along the NW-SE direction: it shows extremely high-velocity red-shifted emission and it is associated with a jet traced by $\mathrm{H}_{2}$ emission as well as with red and blue $\mathrm{H}_{2} \mathrm{O}$ maser spots and $\mathrm{SO}$ emission. Its driving source has to be found in the region associated with the $1.3 \mathrm{~mm}$ object, the $\mathrm{CH}_{3} \mathrm{OH}$ masers, and two massive 
YSOs (VLA 2a and VLA 2b). On the other hand, the extended $\left(\geq 50^{\prime \prime} ; \geq 0.2 \mathrm{pc}\right.$ ) emission along the N-S direction can be separated into at least two more bipolar outflows: one (IRAS 17233-OF2) associated with a counterpart on smaller scales ( $\left.5^{\prime \prime} ; \sim 5000 \mathrm{AU}\right)$ traced by red- and blue-shifted $\mathrm{OH}$ masers and driven by a massive YSO traced by $\mathrm{cm}$-emission (VLA 2c or VLA 2d), and another one (IRAS 17233-OF3) whose driving source has to be found again among VLA $2 \mathrm{a}$ and the $1.3 \mathrm{~mm}$ peak.

3. $\mathrm{H}_{2}$ emission is found mostly in correspondence to the blueshifted clumps, probably due to extinction hiding the redshifted $\mathrm{H}_{2}$ lobes. In two cases, we found extremely high velocity $\mathrm{CO}$ emission associated with $\mathrm{H}_{2}$ emission, suggesting we are observing material accelerated along the jet traced by $\mathrm{H}_{2}$.

4. The molecular outflows were observed also in isotopologues of ${ }^{12} \mathrm{CO}$ : i.e. ${ }^{13} \mathrm{CO}(2-1)$ and $\mathrm{C}^{18} \mathrm{O}(2-1)$, as well as in $\mathrm{SO}\left(6_{5}-5_{4}\right)$. Although covering a smaller velocity range with respect to the ${ }^{12} \mathrm{CO}(2-1)$ line (due to lower line brightness), also in this case the outflow structure is well traced. By comparing the intensity of the ${ }^{13} \mathrm{CO}$ and ${ }^{12} \mathrm{CO}(2-1)$ wings, we have estimated the optical depths: the emission in the ${ }^{13} \mathrm{CO}(2-1)$ line results optically thin, with $\tau_{13} \leq 0.1-0.3$. Consequently, the ${ }^{12} \mathrm{CO}(2-1)$ optical depth (derived simply scaling by the assumed $\left[{ }^{12} \mathrm{CO} /{ }^{13} \mathrm{CO}\right]$ ratio) is quite high ( 10-30), indicating thick emission even at outflow velocities $\pm 30-60 \mathrm{~km} \mathrm{~s}^{-1}$ with respect to the ambient emission.

5. To estimate the relative ${ }^{12} \mathrm{CO}$ excitation conditions of the outflow clumps, we compared the $J=6-5$ and 2-1 line brightnesses. For the blue-shifted lobe the higher the distance of the clump from the driving source(s), the higher is the $R_{62}$ ratio, suggesting higher excitation conditions. In addition, at least for the red clumps, we find indication of an increase of the excitation at the highest velocities, probably closely associated with recently shocked gas.

6. Although based on only two transitions, LVG statisticalequilibrium calculations give a rough estimate of the physical parameters of the molecular gas traced by CO. The red and blue clumps closer to the YSOs are associated with $T_{\text {kin }} \geq 50 \mathrm{~K}$ and with a gas pressure $\geq 10^{6} \mathrm{~K} \mathrm{~cm}^{-3}$.

7. The estimate of the kinematical outflow parameters such as momentum, kinetic energy, and the mechanical luminosity show values that, even taking into account the outflow multiplicity, are typical of massive YSOs $\left(L_{\text {bol }} \geq 10^{4} L_{\odot}\right)$, and in agreement with the measured whole bolometric luminosity of the source $\left(L_{\text {bol }}=2.5 \times 10^{4} L_{\odot}\right)$.

8. The kinematic ages of the flows are in the range $10^{2}-10^{3} \mathrm{yr}$, and therefore point to young objects that still did not reach the main sequence. We compared our results with the scenario described by Beuther \& Shepherd (2005) where jet-like outflows are powered only by young protostars with luminosities that correspond to late B-type main sequence values, and still without an HCHII region. In the case of the $\mathrm{N}-\mathrm{S}$ outflows in IRAS 17233, we observe a high degree of collimation as well as the indication of definitely less collimated wind traced by $\mathrm{OH}$ maser spots. We thus suggest that we are observing a stage where jet-like and less collimated wind co-exist, in agreement with the detection of very small $\left(\sim 10^{-3} \mathrm{pc}\right)$ ionised regions and by the spectral type (B0.5) derived from the outflow parameters.

9. Our analysis shows the importance of high sensitivity observations to detect extremely high velocity emission to access the most energetic part of molecular outflows and detect the counterpart of the $\mathrm{H}_{2}$ emission. Moreover, high-spatial resolution observations of higher excitation $\mathrm{CO}$ lines as well as $\mathrm{SiO}$, sensitive to the hot jet component and not contaminated by ambient gas emission as low rotational CO transitions, would be instructive to better assess the outflow multiplicity in the source and for a better characterisation of the excitation conditions.

Acknowledgements. The Submillimeter Array is a joint project between the Smithsonian Astrophysical Observatory and the Academia Sinica Institute of Astronomy and Astrophysics and is funded by the Smithsonian Institution and the Academia Sinica.

\section{References}

Arce, H. G., Shepherd, D., Gueth, F., et al. 2007, in Protostars and Planets V, ed. B. Reipurth, D. Jewitt, \& K. Keil, 245

Argon, A. L., Reid, M. J., \& Menten, K. M. 2003, ApJ, 593, 925

Bachiller, R., \& Perez Gutierrez, M. 1997, ApJ, 487, L93

Benjamin, R. A., Churchwell, E., Babler, B. L., et al. 2003, PASP, 115, 953

Beuther, H., \& Shepherd, D. 2005, in Cores to Clusters: Star Formation with Next Generation Telescopes, ed. M. S. N. Kumar, M. Tafalla, \& P. Caselli, 105

Beuther, H., Schilke, P., Gueth, F., et al. 2002a, A\&A, 387, 931

Beuther, H., Schilke, P., Sridharan, T. K., et al. 2002b, A\&A, 383, 892

Bonnell, I. A., \& Bate, M. R. 2002, MNRAS, 336, 659

Bonnell, I. A., Bate, M. R., \& Zinnecker, H. 1998, MNRAS, 298, 93

Bronfman, L., Nyman, L.-A., \& May, J. 1996, A\&AS, 115, 81

Caswell, J. L., Haynes, R. F., \& Phys, J. 1980, IAU Circ., 3509, 2

Cesaroni, R., Codella, C., Furuya, R. S., \& Testi, L. 2003, A\&A, 401, 227

Cesaroni, R., Galli, D., Lodato, G., Walmsley, C. M., \& Zhang, Q. 2007, in Protostars and Planets V, ed. B. Reipurth, D. Jewitt, \& K. Keil (Tucson: University of Arizona Press), 951, 197

Codella, C., Bachiller, R., Benedettini, M., et al. 2005, MNRAS, 361, 244

Faúndez, S., Bronfman, L., Garay, G., et al. 2004, A\&A, 426, 97

Fish, V. L., Reid, M. J., Argon, A. L., \& Zheng, X.-W. 2005, ApJS, 160, 220

Fix, J. D., Mutel, R. L., Gaume, R. A., \& Claussen, M. J. 1982, ApJ, 259, 657

Flower, D. R. 2001, J. Phys. B Atom. Mol. Phys., 34, 2731

Forster, J. R., \& Caswell, J. L. 1989, A\&A, 213, 339

Fuente, A., Rodríguez-Franco, A., Testi, L., et al. 2003, ApJ, 598, L39

Hughes, V. A., \& MacLeod, G. C. 1993, AJ, 105, 1495

Krumholz, M. R., \& Bonnell, I. A. 2007 [arXiv: 0712.0828]

Kurtz, S., Cesaroni, R., Churchwell, E., Hofner, P., \& Walmsley, C. M. 2000, Protostars and Planets IV, 299

Lada, C. J. 1985, ARA\&A, 23, 267

Leurini, S., Schilke, P., Parise, B., et al. 2006, A\&A, 454, L83

Leurini, S., Hieret, C., Thorwirth, S., et al. 2008, A\&A, 485, 167

MacLeod, G. C., Scalise, E. J., Saedt, S., Galt, J. A., \& Gaylard, M. J. 1998, AJ, 116, 1897

Marston, A. P., Reach, W. T., Noriega-Crespo, A., et al. 2004, ApJS, 154, 333

Menten, K. M. 1991, ApJ, 380, L75

Miettinen, O., Harju, J., Haikala, L. K., \& Pomrén, C. 2006, A\&A, 460, 721

Natta, A., Grinin, V., \& Mannings, V. 2000, Protostars and Planets IV, 559

Noriega-Crespo, A., Morris, P., Marleau, F. R., et al. 2004, ApJS, 154, 352

Palla, F., \& Stahler, S. W. 1993, ApJ, 418, 414

Preibisch, T., Weigelt, G., \& Zinnecker, H. 2001, in The Formation of Binary Stars, ed. H. Zinnecker, \& R. Mathieu, IAU Symp., 200, 69

Sault, R. J., Teuben, P. J., \& Wright, M. C. H. 1995, in Astronomical Data Analysis Software and Systems IV, ASP Conf. Ser., 77, 433

Schöier, F. L., van der Tak, F. F. S., van Dishoeck, E. F., \& Black, J. H. 2005, A\&A, 432, 369

Scoville, N. Z., Sargent, A. I., Sanders, D. B., et al. 1986, ApJ, 303, 416

van Kempen, T. A., van Dishoeck, E. F., Güsten, R., et al. 2009, A\&A, 501, 633

Walsh, A. J., Burton, M. G., Hyland, A. R., \& Robinson, G. 1998, MNRAS, 301, 640

Wernli, M., Valiron, P., Faure, A., et al. 2006, A\&A, 446, 367

Wilson, T. L., \& Rood, R. 1994, ARA\&A, 32, 191

Xu, Y., Li, J. J., Hachisuka, K., et al. 2008, A\&A, 485, 729

Ybarra, J. E., \& Lada, E. A. 2009, ApJ, 695, L120

Yorke, H. W., \& Sonnhalter, C. 2002, ApJ, 569, 846

Zapata, L. A., Leurini, S., Menten, K. M., et al. 2008, AJ, 136, 1455

Zapata, L. A., Ho, P. T. P., Schilke, P., et al. 2009, ApJ, 698, 1422 